\title{
Time-lapse CSEM: how important is survey repeatability?
}

\author{
Daniil V. Shantsev ${ }^{\oplus},{ }^{1,2}$ Elias A. Nerland ${ }^{1}$ and Leiv-J. Gelius ${ }^{3}$ \\ ${ }^{1}$ EMGS, Imaging \& Interpretation, Karenslyst Allè 4, N-0278 Oslo, Oslo, Norway.E-mail: shantsev@gmail.com \\ ${ }^{2}$ University of Oslo, Department of Physics, Problemveien 7, 0315 Oslo, Oslo, Norway \\ ${ }^{3}$ University of Oslo, Department of Geosciences, Problemveien 7, 0315 Oslo, Oslo, Norway
}

Accepted 2020 September 18. Received 2020 September 16; in original form 2019 November 10

\begin{abstract}
SUMMAR Y
An important concern for time-lapse studies using the controlled-source electromagnetic (CSEM) method is repeatability of acquisition parameters for the base and monitoring surveys. We consider a challenging case when variations in source and receiver positions, conductivity of seawater, etc. will lead to differences in the recorded EM fields that exceed EM response due to production-induced changes in the reservoir resistivity. We show that even in that case, 4-D response can often be clearly resolved if the base and monitor EM data sets are inverted to produce 3-D resistivity distributions. More precisely, for a synthetic model of the Snøhvit gas field, changes in the inverted resistivity maps caused by such non-repeatability will be at least 10 times smaller than the time-lapse differences in the reservoir resistivity. By contrast, measurement errors and poor knowledge of background resistivity may reduce the resolution of 4-D CSEM to a much stronger degree. Analysis of field CSEM data from the Wisting oil field supports our conclusion about strongly relaxed repeatability requirements when 4-D effects are established by examining inverted resistivity volumes rather than by looking at raw EM data.
\end{abstract}

Key words: Controlled source electromagnetics (CSEM); Marine electromagnetics.

\section{INTRODUCTION}

The controlled-source electromagnetic (CSEM) method is frequently used to provide information about 3-D resistivity distribution in the subsurface, and hence about rock porosity and fluid saturation (Constable 2010; Streich 2016). CSEM surveys have often been applied for hydrocarbon exploration - to help making drilling decisions and finding new targets (see e.g. Alcocer et al. 2013; Løseth et al. 2014), as well as for imaging $\mathrm{CO}_{2}$ plume at carbon storage sites (Park et al. 2017). Recent years have seen a significant progress in various aspects of marine CSEM technology: launch of a new source with up to $10000 \mathrm{~A}$ electrical current (Hanssen et al. 2017), pushing acquisition frequencies up to $45 \mathrm{~Hz}$ to reach a record high spatial resolution (Granli et al. 2018) and a common use of advanced Gauss-Newton optimization schemes for large-scale 3-D inversions (Nguyen et al. 2016; Perez et al. 2020).

There is a great potential also in time-lapse applications of CSEM. It can allow monitoring pore fluid during production of hydrocarbon reservoirs - by imaging the corresponding changes in electric resistivity. 4-D CSEM can be especially useful in situations when 4-D seismic cannot give all the answers. For example, seismic methods struggle to discriminate between changes in pore pressure and saturation (Landrø 2001) and adding CSEM data that has sensitivity mostly to saturation can resolve this problem (Wang \& Gelius 2010; Salako et al. 2015). Besides, CSEM has maximum sensitivity at high hydrocarbon saturations, allowing for early detection of fluid flow patterns under production. By contrast, the acoustic impedance does not change much until the saturation drops to low values. Finally, it is often difficult to discriminate from 4-D seismic data between heavy oil and brine, while CSEM data will show maximum contrast between these two fluids due to their vastly different resistivity values. 4-D CSEM can also be applied for monitoring of $\mathrm{CO}_{2}$ injection, as suggested by field (Girard et al. 2011, boreholeto-surface setup) and synthetic (Bhuyian et al. 2012; Zhdanov et al. 2013; Kang et al. 2015) studies.

Numerous synthetic studies have shown that CSEM sensitivity can be sufficiently high to resolve small resistivity changes in various monitoring scenarios. Some of these studies are based on synthetic models of existing producing hydrocarbon fields (Ziolkowski et al. 2010; Black et al. 2011; Colombo \& McNeice 2013; Zerilli et al. 2018). Some studies use reservoir simulations to demonstrate that CSEM data can be directly linked to monitoring changes in the fluid saturation and motion of the flooding front (Ziolkowski et al. 2010; Liang et al. 2012; Shahin et al. 2012; Zerilli et al. 2018). Other papers (Lien \& Mannseth 2008; Wirianto et al. 2010) show how one can deal with typical challenges of 4-D CSEM analysis such as noise, measurement and modelling errors, by means of for example optimizing acquisition geometry.

Despite abundance of theoretical studies, there have been almost no marine CSEM surveys aiming specifically at time-lapse applications. The only exception we are aware of is the transient EM surveys in 2007 and 2008 along one line over the Harding oil and 
gas field (Ziolkowski et al. 2010). One of the main obstacles towards practical use of 4-D CSEM seems to be the repeatability concerns between the base and monitoring surveys, especially in a marine environment. Indeed, it is essentially impossible to ensure precise repeatability of acquisition parameters such as position and orientation of source and receivers, conductivity and depth of seawater, source current and receiver calibration.

Several papers have specifically addressed repeatability requirements for 4-D CSEM (Orange et al. 2009; Zach et al. 2009; Wirianto et al. 2010; Andreis \& MacGregor 2011; Tietze et al. 2015, 2019). All their analyses have been performed in the data domain, that is assumed that EM data from the base- and monitoring surveys will be compared directly. Hence, differences in the recorded EM fields induced by non-repeatability of survey parameters should be smaller than the 4-D response-differences in EM fields due to changes in the reservoir resistivity. This condition is difficult to fulfill in practice, as argued by Orange et al. (2009) and Andreis \& MacGregor (2011) who concluded that permanent seafloor installations might be needed to make 4-D CSEM feasible. Fortunately, even if these strict repeatability requirements derived in the data-domain approach are violated, it does not necessarily mean that 4-D CSEM is unfeasible, as demonstrated in the present work.

Repeatability requirements can be significantly relaxed if analysis of 4-D effects is performed in the model domain rather than in the data domain. In other words, instead of comparing the two measured CSEM data sets, one can invert them and compare the resulting resistivity models. Acquisition parameters are a part of the inversion setup for each data set and it is expected that their non-repeatability will not directly impact time-lapse differences in the inverted resistivity distributions. This expectation has already been voiced earlier for CSEM (see e.g. Andreis \& MacGregor 2011). Besides, there exist a few other studies that used inversion of CSEM data to determine 4-D responses in the model domain (Ziolkowski et al. 2010; Black et al. 2011; Liang et al. 2012; Colombo \& McNeice 2013; Zhdanov et al. 2013; Kang et al. 2015; Patzer et al. 2017). However, none of them investigated the effect of acquisition non-repeatability on the quality of the inverted 4-D response. Zerilli et al. (2018) state that 'strong' non-repeatability was mitigated in their inversions but gives no quantitative measure of non-repeatability 'strength' or its effect on inversion results. The aim of the present work is to fill the existing gap and establish new repeatability requirements for 4-D CSEM, applicable when 4-D effects are analysed in the model domain. It is interesting to note that when 4-D seismic data is analysed using inversion rather than conventional seismic processing, it also leads to introduction of more relaxed repeatability requirements for 4-D seismic surveys (Qu \& Verschuur 2017).

Preliminary results on the repeatability requirements for inversion-based 4-D CSEM were presented in our earlier works (Babakhani 2015; Shantsev et al. 2018). They considered nonrepeatability in water conductivity and receiver positions but used a low-dimensional (2.5-D) approach and simplistic synthetic model with a uniform background. Here we extend this analysis by going to 3-D, using a geological model representing the producing Snøhvit gas field in the Barents Sea, and consider more types of nonrepeatability. The standard sensitivity analysis, and the inversionbased approach are shown to produce dramatically different results in terms of repeatability requirements. In the last section we analyse field CSEM data acquired near the Wisting oil discovery in order to test conclusions from the synthetic study.

The main focus of the present synthetic study is on the repeatability requirements rather than on the feasibility of 4-D CSEM in general. Therefore we limited ourselves to somewhat simple choices about background resistivity, reservoir size, depletion pattern and survey layout. A detailed analysis of these factors on the resolution of 4-D CSEM would require a separate study based on more sophisticated models.

\section{SNØHVIT MODEL}

Snøhvit is a producing oil- and gas field located in the Hammerfest basin in the southwestern part of the Norwegian Barents Sea. The Snøhvit discovery was made in 1994 by the 7121/4-1 exploration well. The greater Snøhvit field includes the Snøhvit, Albatross- and Askeladden structures (Fig. 1). Snøhvit was put into production in 2007 and produces gas and condensate from the early- and midJurassic Nordmela and Stø formations. A cross section through the model is given in Fig. 1(a). The reservoir is located at $2300 \mathrm{~m}$ depth (TVD) with water depths of 310-340 m.

Multiclient 3-D CSEM data have already been acquired over large parts of the Snøhvit structure. 3-D inversion of these data has proven that the Snøhvit discovery can be detected as a clear resistive anomaly (Gabrielsen et al. 2012). Meanwhile, the CSEM sensitivity to the target is not very high due to relatively large burial depth and high background resistivities. All this makes the Snøhvit field a good practical example for a 4-D synthetic study.

We have built a 3-D synthetic model of the area based on public information and EMGS' inversion experiences from the available multiclient surveys in the Hammerfest basin. The background resistivity was derived from well logs and CSEM inversion results. The vertical resistivity values lie between 7 and $18 \Omega \mathrm{m}$, see a cross section in Fig. 1(a) while the horizontal resistivity values lie between 1.75 and $6 \Omega \mathrm{m}$. A target resistor has been placed in the model with a lateral extension following the Snøhvit outline given by the Norwegian Petroleum Directorate (NPD). The target base level was chosen to be at $2425 \mathrm{~m}$, which approximately corresponds to the gas-il contact in the upper reservoir found from RFT (Repeat Formation Tester). The oil zone, 2425 to $2442 \mathrm{~m}$ in the Nordmela Formation, consists of an interbedded sandstone/shale sequence with fair/poor reservoir properties. For simplicity, we focus on the upper gas filled reservoir and analyse 4-D effects only in that part. The gross reservoir thickness was calculated from Top Stø to the assumed contact at $2425 \mathrm{~m}$ for the base case and varying contacts for monitoring cases (see Fig. 1d).

The key parameter of a hydrocarbon reservoir that determines its CSEM response is ATR-nomalous Transverse Resistance (see e.g. Baltar \& Roth 2013; Gabrielsen et al. 2013). It is essentially a product of the target thickness and a difference of the target and background resistivities. To prepare a synthetic Snøhvit resistivity model, we first compute the lateral distribution of the target's ATR. The measured reservoir resistivity peaks above $2000 \Omega \mathrm{m}$, but the average anomalous reservoir resistivity is much lower, and we roughly estimated it to be $160 \Omega \mathrm{m}$ across the gross reservoir interval. Thus, ATR maps were obtained by multiplying $160 \Omega \mathrm{m}$ with the gross thickness maps.XXX

We assume a production scenario where the gas-oil contact rises uniformly throughout the reservoir, starting from $2425 \mathrm{~m}$ towards 2412.5, 2400 and $2375 \mathrm{~m}$ for the three monitoring stages. ATR maps for the three different positions of the contact are shown in Fig. 1(d). The target was imprinted into the background resistivity models by increasing resistivity within a 720 -m-thick layer by a laterally varying value that ensures a precise match to the presented 


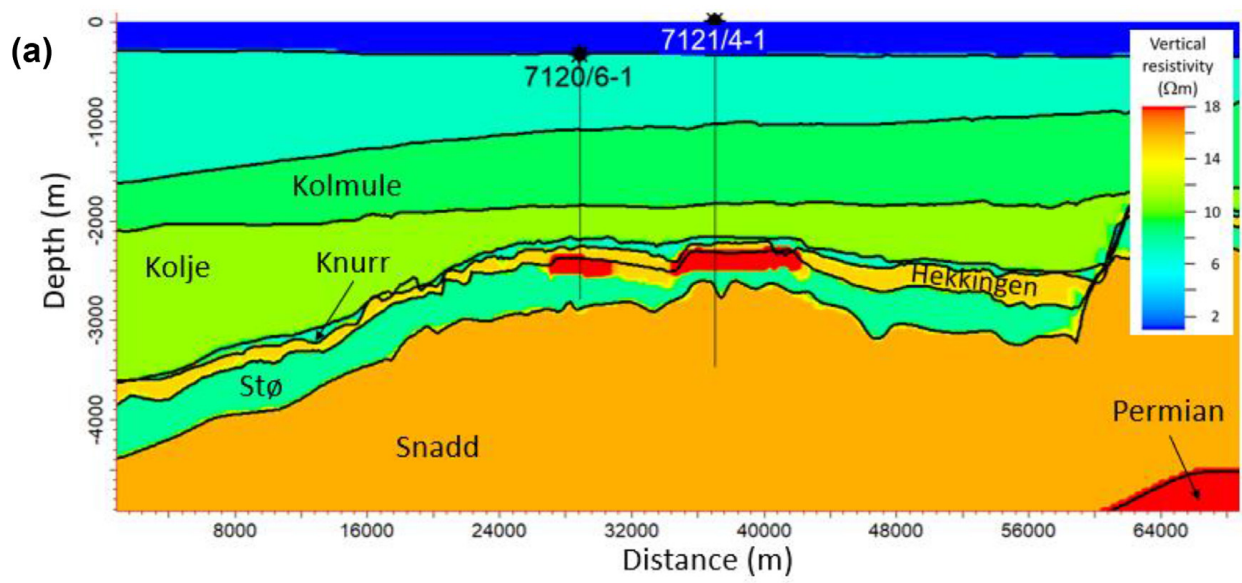

(b)
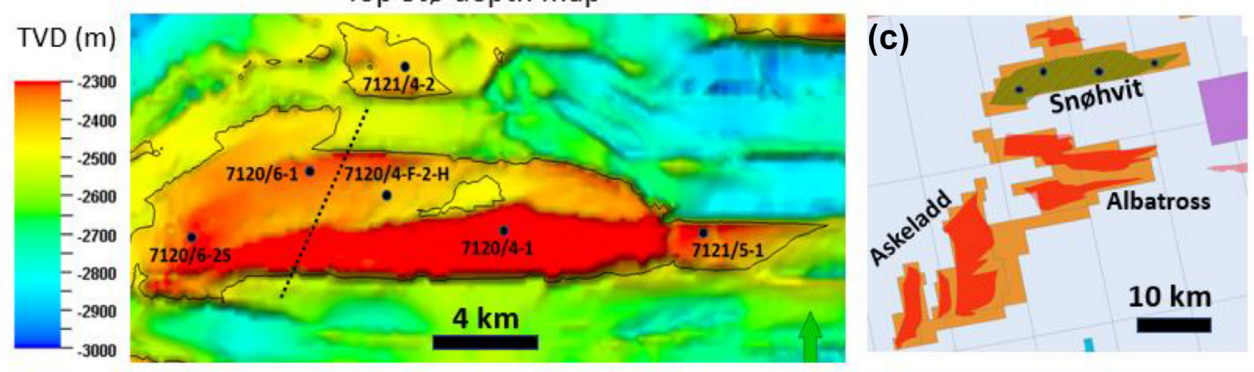

(d)

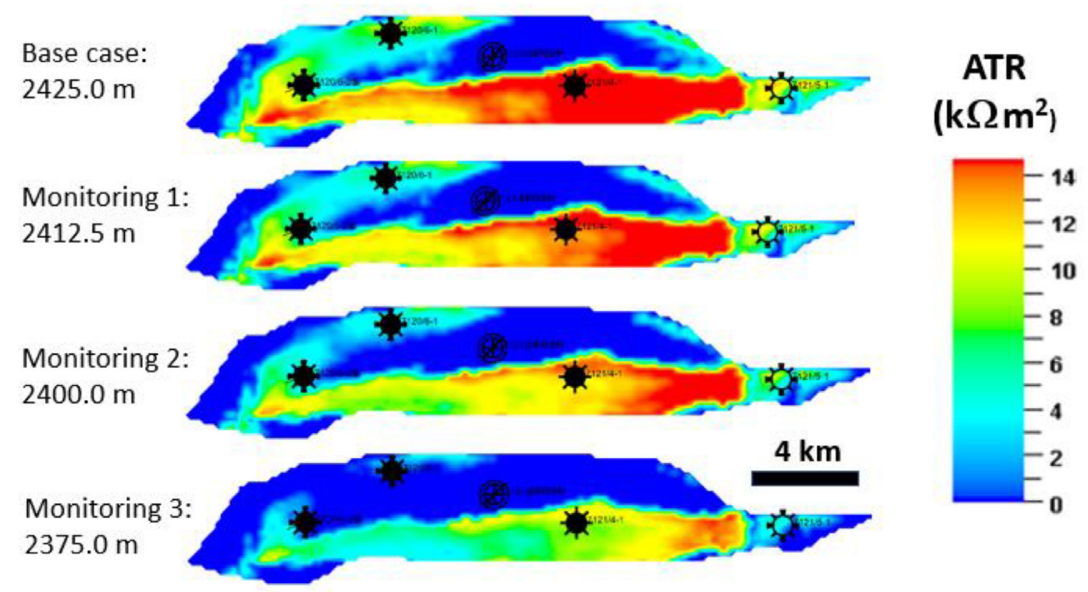

Figure 1. (a) Vertical resistivity cross section through the Snøhvit model. The layers have uniform resistivity, layer boundaries are provided by Bow Geo. (b) Top Stø depth map; dashed line - an artificial internal barrier introduced in the model to allow for different production scenarios. (c) Overview map of 'Greater Snøhvit' area. The modelling is carried out for the main Snøhvit unit which is currently producing. (d) Imprinted ATR for different production stages, with indicated contact depth. As the contact is lifted, the ATR is reduced evenly throughout the reservoir.

ATR maps (one could have imprinted the target as a layer of constant resistivity and varying thickness; it would have been a more geologically sound approach, but less practical because of finite vertical size of model cells). ATR decrease during production was thus modelled via reduction of resistivity. The maximum resistivity over the reservoir went down from $157 \Omega \mathrm{m}$ before production to 145,133 and $108 \Omega \mathrm{m}$, respectively, for the three monitoring stages.

In the main production scenario, it is assumed that full communication is existing within the reservoir without any internal barriers. An alternative scenario has been modelled where an internal barrier was introduced to simulate the presence of a sealing fault (Fig. 1b). This barrier is purely artificial and is not based on any interpretation of the Snøhvit reservoir. The motivation for introducing a barrier in the CSEM time lapse experiment is to evaluate whether lateral resolution allows for detecting the compartmentalization within such a reservoir. In the alternative scenario it is assumed that all production is taking place east of the barrier while in the west the virgin conditions remain through production history and hence ATR does not change. The impact of non-repeatability that we aim to evaluate probably should not critically depend on the complexity of the flooding pattern, but more research into this would be appreciated. 


\section{CSEM DATA}

Fig. 2 shows an outline of a synthetic CSEM survey over the Snøhvit target. The survey consists of 6 parallel towlines spaced by $1 \mathrm{~km}$ and a grid of 57 seabed receivers spaced by $3 \mathrm{~km}$ along the towing direction. This is a relatively sparse survey as could be seen, for example by comparing it to a recent CSEM acquisition over the Gemini North discovery in the Barents Sea where the receiver spacing was $1 \mathrm{~km}$ and the towline spacing-500 $\mathrm{m}$ (Granli et al. 2018). The present study therefore does not aim at probing the resolution limit for 4-D CSEM that would have required a much denser survey. The focus of this work is solely on non-repeatability effects.

The recorded data include horizontal components of both electric $\boldsymbol{E}$ and magnetic $\boldsymbol{H}$ fields at frequencies $0.2,0.4,0.6$ and $1 \mathrm{~Hz}$. The source is a horizontal electric dipole towed $30 \mathrm{~m}$ above the seabed, the source current is directed along the towing direction (the $\boldsymbol{x}$ axis). We consider full-azimuth data, that is a receiver is active for the source located at the inline towline as well as at azimuthal towlines. The maximal source-receiver offset is chosen to be $15 \mathrm{~km}$, the shot point spacing is $100 \mathrm{~m}$. When inverting the CSEM data, the shortest offsets, less than $2 \mathrm{~km}$, were excluded from the analysis. This is a standard measure since EM fields rapidly decay at short offsets and even small measurement errors in source or receiver positions lead to significant distortions of EM signal.

The synthetic CSEM data has been generated for the base case and for all the monitoring cases described above. The differences between them characterize the sensitivity of CSEM data to the reservoir production effects. These differences $\left|\boldsymbol{E}_{\text {base }}-\boldsymbol{E}_{\text {mon }}\right|$ for the electric field were normalized by data uncertainty and plotted in Fig. 3(a), while the corresponding differences for magnetic field are shown in Fig. 3(b). They were computed for a receiver marked by a circle in Fig. 2, and include only inline source positions (towline Tx004). The value of one indicated by dashed lines is where the field difference equals the uncertainty. The total data uncertainty was computed by assuming 1 per centXXX multiplicative noise and a noise floor of $10^{-16} \mathrm{~V} \mathrm{Am}^{-2}$ for $\boldsymbol{E}$ and $10^{-13} \mathrm{~m}^{-2}$ for $\boldsymbol{H}$ fields. For monitoring stages 2 and 3 the curves go above one at long offsets, that is sensitivity to 4-D effects is larger than the data uncertainty. Nevertheless, the observed sensitivity values are still pretty lowbetween 1 and 3-implying that detecting 4-D effects in this case is challenging. Note that sensitivity in magnetic data (b) is at least as large as in the electric data (a), therefore magnetic data is equally valuable for the analysis.

The bottom of Fig. 3 shows a response map constructed using data for all receivers and a common midpoint (CMP) approximation. We chose the offset of $13 \mathrm{~km}$ that gives the maximal response according to Fig. 3a. The map is computed for monitoring stage 2, for full azimuth electric field data at $0.2 \mathrm{~Hz}$, and the black dots indicate the receiver locations. The maximum response in the map is around 2.0, which is close to the peak value 1.75 for the selected receiver in Fig. 3a. In the CMP approximation, the response is attributed to the location in the middle between the source and receiver positions. Indeed, the red zone of the maximal response lies between the selected receiver (marked by a circle in Fig. 3c), and the corresponding source position $13 \mathrm{~km}$ to the left. It is interesting to compare this 4-D response map and Fig. 1d showing ATR maps for the base and monitoring models. ATR values are lower in the left half of reservoir, hence a homogeneous production would result in larger relative ATR changes in the left half. This is probably why we see there the largest 4-D response in the electric field in Fig. 3c.

The uncertainty level of 1 per cent assumed for this study, as well as the chosen noise floors are the high-end values, see for example uncertainty analysis in Mittet \& Morten (2012). This choice comes from the natural assumption that in 4-D CSEM surveys aiming at finer resolution than the standard 3-D CSEM, a special care will be taken to push for the lowest possible noise levels. CSEM equipment that may allow reaching these low levels has recently been demonstrated in Hanssen et al. (2017).

\section{INVERTING TIME-LAPSE RESPONSE}

Let us now run 3-D inversion of the synthetic CSEM data for different monitoring stages. Prior to inversion, the data were contaminated with noise using the same noise model as described above for the data uncertainty. Namely, we added 1 per cent uncorrelated Gaussian multiplicative noise for each data point and an uncorrelated additive Gaussian noise corresponding to noise floor of $10^{-16} \mathrm{~V} / \mathrm{Am}^{2}$ for $\boldsymbol{E}$ and $10^{-13} \mathrm{~m}^{-2}$ for $\boldsymbol{H}$ fields. The 3-D inversion was based on a quasi-Newton BFGS optimization algorithm described in Zach et al. (2008) and typically took less than 30 iterations. A conventional smoothness regularization with an L2 norm was used for the logarithm of the resistivity values.

During production it is expected that the resistivity is changing only in the reservoir area, while the background resistivity remains constant. Even if the reservoir geometry is not known precisely, it is usually easy to outline its maximal possible extent and postulate that the resistivity is not changing beyond this outline. In the present study the inversions were allowed to update resistivities only within the volume of the actual reservoir, while the background resistivity always remained unchanged. As a result, the inversion could effectively focus only on a relatively small region of the whole computational domain, which doesn't only make the inversion more efficient, but also helps a lot with imaging the reservoir accurately.

The resistivity model was discretized on a regular grid with $200 \mathrm{~m}$ cell size in lateral direction and $40 \mathrm{~m}$ in depth. The reservoir volume thus included 6691 cells, each of them having two free inversion parameters: the vertical and horizontal resistivities. The inversion was not allowed to reduce resistivities below 80 per cent of their background values in the absence of hydrocarbons or increase them above $200 \Omega \mathrm{m}$ (which is well above the maximal resistivity in the base model). The forward problem was solved on a rectilinear nonuniform grid with the vertical cell size being $40 \mathrm{~m}$ down to $2800 \mathrm{~m}$ depth and slowly growing for deeper levels, and the horizontal cell size varying from $200 \mathrm{~m}$ close to the EM source to $400 \mathrm{~m}$ at $15 \mathrm{~km}$ offset, with perfectly matched layers (PMLs) on top of that.

The standard inversion convergence criterion is based on the data misfit. For the present 4-D study, where tiny resistivity changes need to be resolved, we added an additional criterion based on the inverted resistivity. At each inversion iteration, the average resistivity over the reservoir was computed and the inversion was allowed to stop only if this resistivity also converged. More specifically, relative differences between average resistivities for several subsequent iterations could not exceed $10^{-4}$. In addition, a similar criterion was used for the data misfit, with maximal relative difference being $10^{-3}$. Finally, it was required for convergence that the data misfit normalized to uncertainty should drop below one (usually it converged to 0.6-0.7).

An important remaining question is how to determine the background resistivity. The most promising approach is to find it using simultaneous inversion of the base and monitoring data sets that should both correspond to the same background. In this work we limit ourselves to the standard inversions based on one CSEM data set only. First, we consider the most optimistic case-the precise knowledge of the background, and later in the paper investigate the 


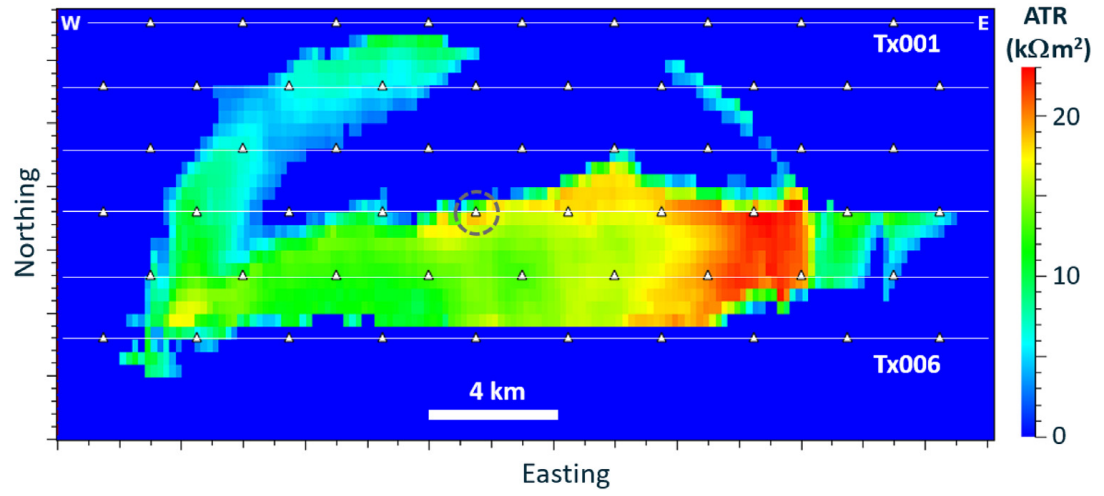

Figure 2. An outline of a synthetic CSEM survey overlaid by anomalous transverse resistance of the Snøhvit target. The survey consists of 57 seabed receivers spaced by $3 \mathrm{~km}$ along towlines, the towline spacing is $1 \mathrm{~km}$ (scaling in Easting is compressed relative to scaling in Northing).
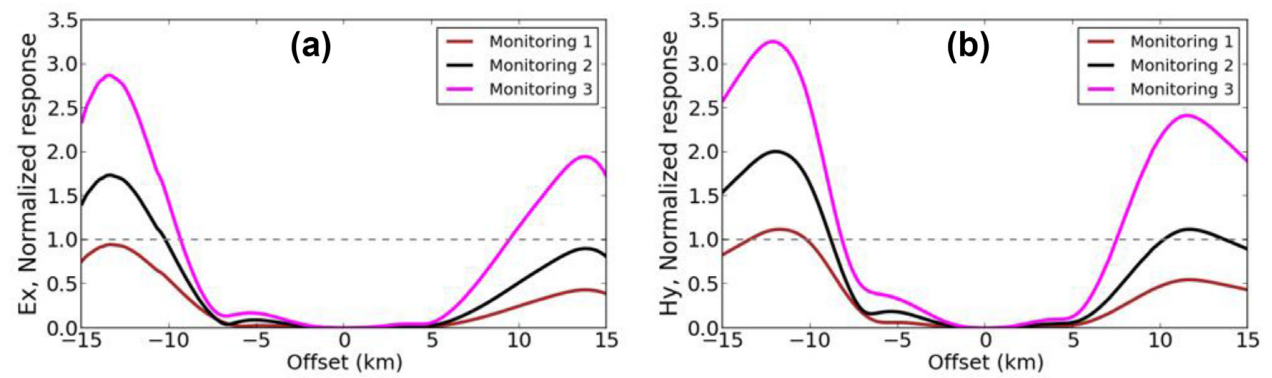

(c)

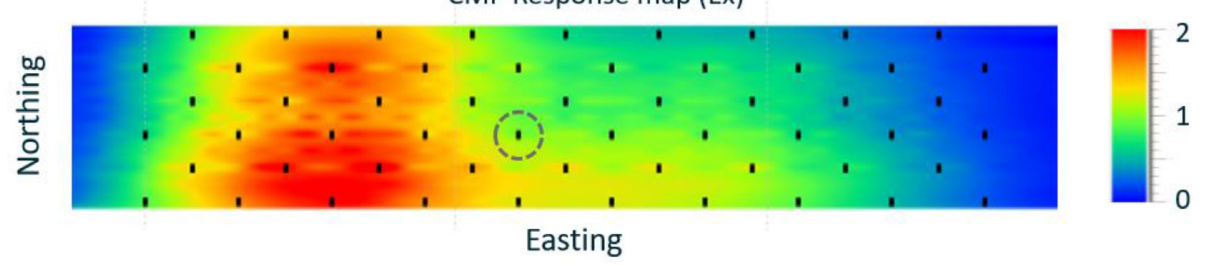

Figure 3. Sensitivity of CSEM data to production-induced changes in resistivity, at frequency $0.2 \mathrm{~Hz}$. Top: Electric (a) and magnetic (b) field data for receiver marked in (c) and also in Fig. 2. Dashed line: sensitivity threshold when the difference between the base and monitoring data equals the data uncertainty. Bottom (c): CMP response map for all receivers, full-azimuth electric data, offset $13 \mathrm{~km}$, monitoring phase 2 . The black dots indicate receivers. The maximal response is approximately 2 and located mostly in the left half of the reservoir.

effect of imprecise background resistivity. The assumption of perfect knowledge of background resistivity was also adopted in earlier 4-D CSEM studies, for example by Colombo \& McNeice (2013) and Patzer et al. (2017).

The resistivity in the reservoir area in the start model was set to the background resistivity of the same layer. In other words, it was assumed that there were no hydrocarbons there. Once the inversion starts running, it is building up the reservoir resistivity until the data misfit becomes small enough. Inverting the monitoring data sets converged to slightly lower resistivity values in the reservoir than inverting the base data set, as expected. In the 4-D scenario, one is interested in differences between the base- and monitoring cases, and they are presented as ATR-maps in Fig. 4. These ATRmaps show production-induced changes in transverse resistance, that is the difference in resistivities, $\boldsymbol{R}_{\text {base }}-\boldsymbol{R}_{\text {monit }}$, integrated over the depth interval that covers the whole hydrocarbon reservoir:

$$
\mathrm{A} T R=\int\left(\boldsymbol{R}_{\text {Base }}-\boldsymbol{R}_{\text {Monit }}\right) d z
$$

Note that from now on we shall use the abbreviation ATR, anomalous transverse resistance, in the 4-D sense, that is our reference case will be the base survey, and ATR will characterize a reduction in transverse resistance due to reservoir production.

The left half of Fig. 4 shows the "true" ATR maps, while the right part shows ATR maps obtained by inverting CSEM data. The bottom row corresponds to the first monitoring stage, where the production induced changes in resistivity are smallest, while the top row corresponds to the monitoring stage 3 , with largest resistivity changes. The inversion results reproduce these trends: the differences in inverted resistivity are larger for later production stages, and their magnitudes are not far from magnitudes in the "true" resistivity maps. There is no perfect match between the "true" and inverted resistivity difference maps. The resistivity change averaged over the reservoir volume is not perfect either, for example for the second monitoring stage it is $\sim 22 \Omega \mathrm{m}$ for the "true" models, but only $\sim 17 \Omega \mathrm{m}$ for the inverted models. Nevertheless, this level of agreement can be considered reasonably good for this challenging case where CSEM data sensitivity is of the order of the noise level, as indicated by Fig. 3 .

Both production scenarios - with and without a sealing fault - are presented in Fig. 4. With the sealing fault in place, the production occurs only to the right of the fault and the resistivity changes are 
$\underline{\text { True }}$
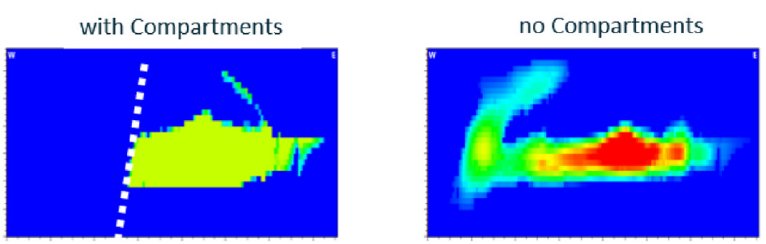

Inverted
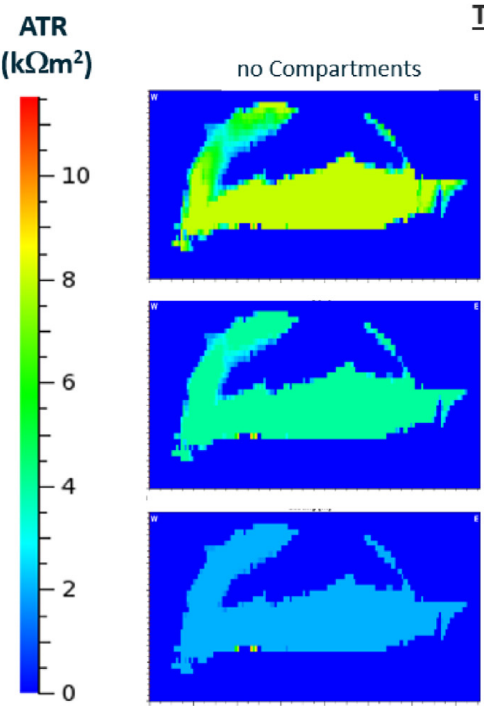

$\left(\mathrm{k} \Omega \mathrm{m}^{2}\right)$

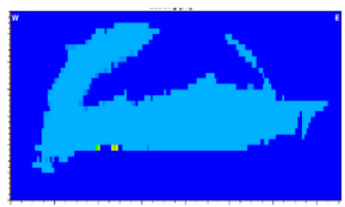

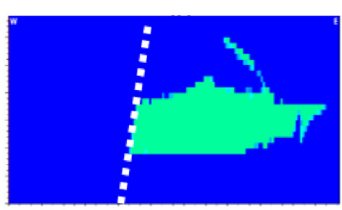

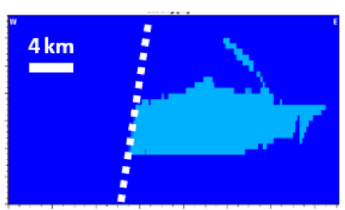

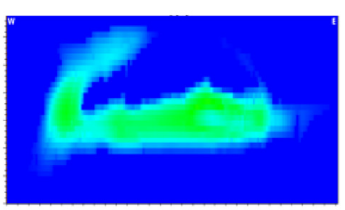

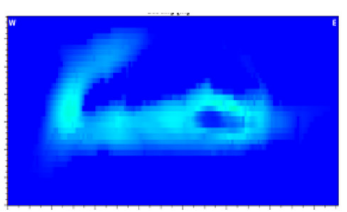

with Compartments
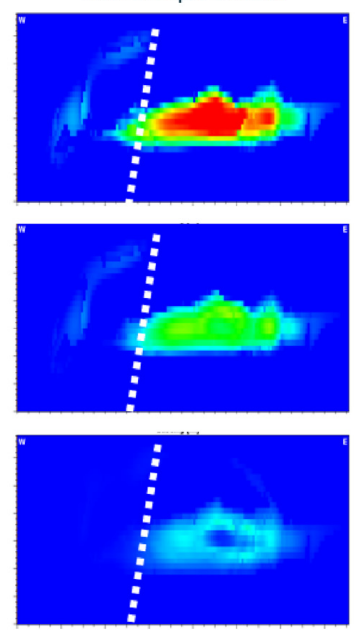

Figure 4. Differences in transverse resistance between the base and monitoring surveys. Survey parameters were identical. Left: "true" differences used to generate CSEM data. Right: differences obtained by inverting these data. Monitoring stages 1, 2 and 3 correspond to the bottom, middle and top rows, respectively. The inverted maps are in fair agreement with "true" maps and allow one to discriminate between different production scenarios (with and without compartmentalization).

located only in that part of reservoir. The inversion results for these two cases indeed reproduce the expected behavior. In the presence of a sealing fault, we observe almost no resistivity changes in the left part, except for some tiny variations that cannot be avoided since CSEM data were contaminated with noise. Hence CSEM data would allow one to clearly distinguish which of the two scenarios takes place, even at the early production stages. In the rest of the paper we show results only for the production scenario with uniform flooding (without compartments).

So far it has been assumed that the base and monitoring surveys had identical acquisition parameters, but in the next sections we shall focus on the main topic of this study: effect of their nonrepeatability.

\section{NON-REPEATA BILITY: DATA-DOMA IN}

The base and monitoring surveys may be acquired with a time interval of several years, and thus their acquisition parameters cannot be reproduced precisely. In previous studies considering feasibility of 4-D marine CSEM, authors analysed non-repeatability of seawater conductivity, source and receiver position and orientation, source waveform and near-surface inhomogeneities (Orange et al. 2009; Zach et al. 2009). In the present study we consider non-repeatability of seawater conductivity, source depth and pitch and receiver position at the seabed.

Seawater conductivity depends on salinity and temperature and we assume that it varies the same way as in the Orange et al. (2009) study: from 3.03 to $3.175 \mathrm{~S} \mathrm{~m}^{-1}$ for the base and monitoring surveys, that amounts to a variation of $\sim 4.5$ per cent. This variation impacts the measured CSEM data, which is illustrated in Fig. 5a where we show the corresponding differences in the inline $\boldsymbol{E}$ field. The difference has a peak at shorter offsets, due to EM signal travelling through the water and reflecting from the sea surface, and another peak at longer offsets due to the air-wave, see Orange et al. (2009) for a more detailed discussion. The differences are quite significant: they are at least twice the sensitivity level for all frequencies and offsets; and up to 6-8 times larger at the peaks.
The standard CSEM source is a $\sim 300 \mathrm{~m}$ long horizontal antenna towed about $30 \mathrm{~m}$ above seabed. The antenna path depends on the vessel speed and water currents and cannot be reproduced precisely when repeating towing. We have assumed that the difference in antenna depth for the base- and monitoring surveys is a random function of coordinate and its rms equals $10 \mathrm{~m}$, see Fig. 5c. Since the antenna path must be smooth, a correlation length of the depth variation was assumed to be a few kilometers, similar to an earlier work (Shantsev et al. 2012). Source depth variations must be accompanied by variations in the source pitch, thus we introduced these too, with the same correlation length and rms pitch difference of 3 degrees. The combined impact of non-repeatability in source depth and pitch on the CSEM data is shown in Fig. 5b. It turned out that the dominant contribution comes from the depth variations, and there exists a clear correlation between the peaks in Fig. $5 \mathrm{~b}$ and depth profiles in Fig. 5c. Namely, the strongest peaks are observed where the difference in source depth was maximal: at offsets -10.5 , $-7,9$ and $12 \mathrm{~km}$. Moreover, the peaks are weaker for lower frequencies since they experience less attenuation while travelling several additional meters through the seawater. The peak magnitude is quite large again, up to factor of 8 or more above the sensitivity threshold.

Finally, it is assumed that receivers land at different positions at the seabed for the two surveys. It is hardly possible to reproduce their position exactly because one never has a perfect knowledge of water currents that make receivers drift away when falling through the water layer. It was assumed that the variations in receiver positions between the two surveys follow a Gaussian distribution with an average shift being $50 \mathrm{~m}$ in each lateral direction. Note that for a realistic 4-D acquisition it is usually possible to repeat receiver positions with a better accuracy. However, our goal here is to demonstrate that even for large receiver displacements, the effect of this non-repeatability on inverted resistivity is not as strong as one might expect. As a reference point, one can mention that for the 2007-2008 surveys using ocean-bottom-cable technology the repeatability was on the order of $10 \mathrm{~m}$ (Ziolkowski et al. 2010).

The top right panel of Fig. 5 shows how the inline electric field is affected by receiver displacement. Data in this figure correspond 

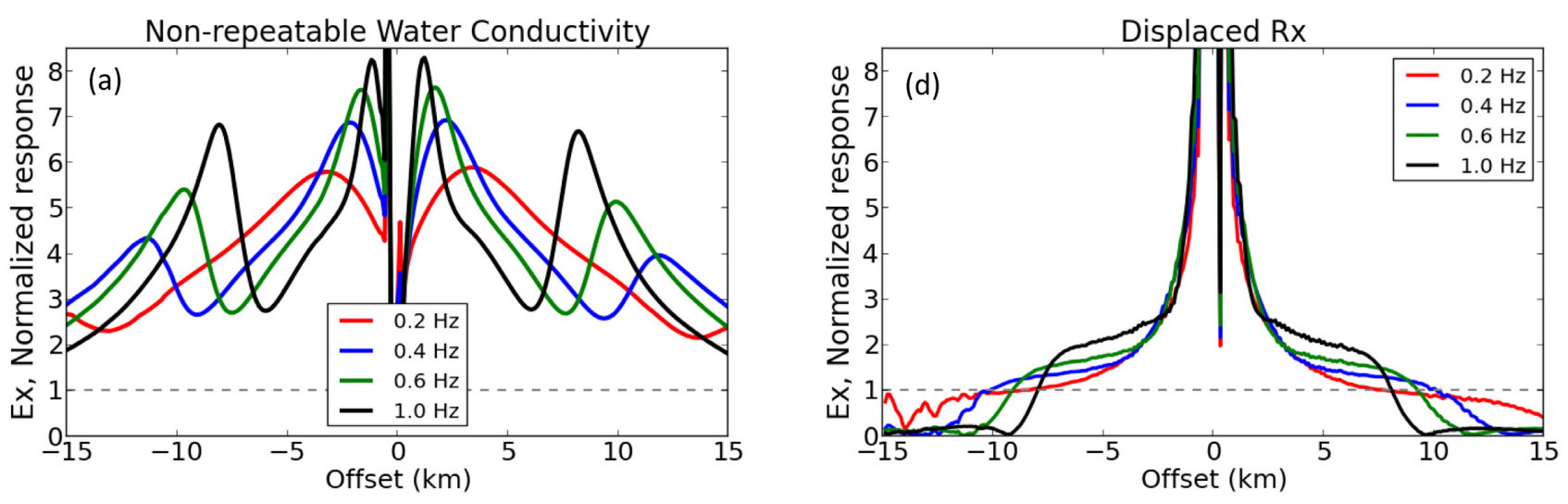

Non-repeatable Tx depth \& pitch
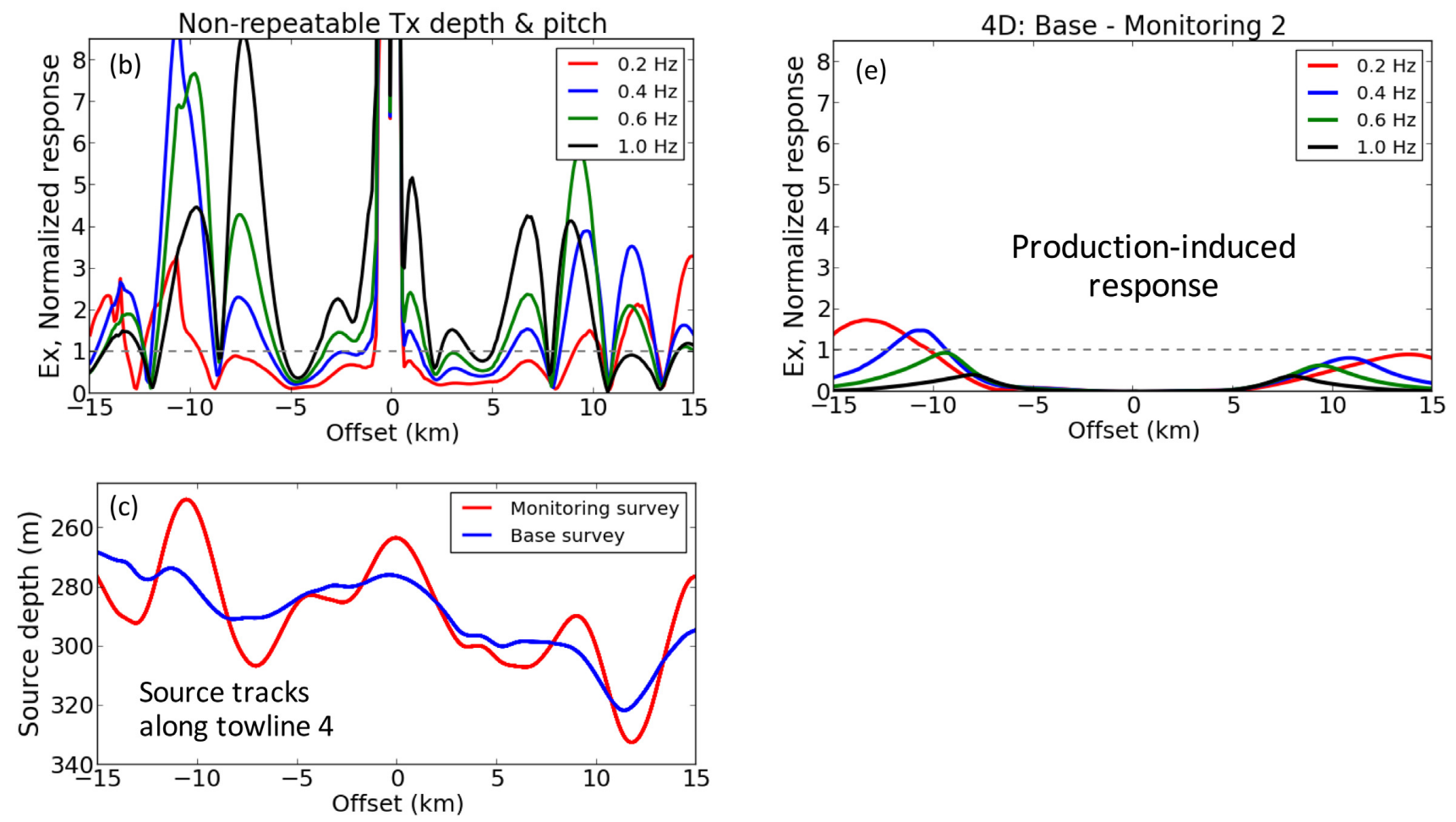

Figure 5. Effects of non-repeatability in acquisition parameters: seawater conductivity (a), source depth and pitch (b), and receiver position(d) on CSEM data. Each of them is much stronger than the 4-D effect due to reservoir production (e) for monitoring stage 2 implying that 4-D effects could not be detected if they are analysed in the data domain. Panel (c) shows source depth traces for two surveys: largest deviations in depth correlate well with peaks in (b). All data here correspond to receiver marked by circle in Fig. 2 and inline towline Tx004. Electric field response is normalized to data uncertainty.

to a receiver that was shifted by only $20 \mathrm{~m}$ along towline and $16 \mathrm{~m}$ in the cross-line direction, that is much smaller than the average displacement of $50 \mathrm{~m}$. Nevertheless, the impact on data is quite significant up to $\sim 10 \mathrm{~km}$ offsets. The impact is maximal at short offsets and decays towards longer offsets because it is roughly proportional to the spatial derivative of the EM field.

It is important now to compare the effect of non-repeatability on CSEM data illustrated in the panels $(a, b, d)$ of Fig. 5 and its panel (e) that shows the 4-D effect from reservoir production (for monitoring stage 2). Obviously, the production induced variation of resistivity in the reservoir has a much smaller impact on the CSEM data. It is close to the sensitivity limit, as we have already seen in Fig. 3. Comparing different panels on Fig. 5 it is tempting to conclude that 4-D effects cannot be resolved here since they will be masked by much stronger non-repeatability effects. Indeed, this would be the case if 4-D effects are analysed in the data domain, that is by comparing the measured EM fields. Random variations of acquisition parameters will result in significant differences in the measured fields between the base and monitoring surveys. These differences will have a complicated frequency- and offset dependence, and it will seem impossible to detect a weak 4-D response due to reservoir production on top of that. Fortunately, we do not have to detect 4-D effects by looking directly at the CSEM data: one can run a CSEM inversion instead, which may lead to very different conclusions about the importance of non-repeatability, as shown in the next sections.

\section{NON-REPEATABILITY: MODEL-DOMAIN}

Like in the previous section, it is assumed that some acquisition parameters have changed from the base to the monitoring survey. 
Namely, the seawater conductivity uniformly changed by $\sim 4.5$ per cent, each receiver was shifted laterally by a random distance that on average makes $50 \mathrm{~m}$ in each direction, the source depth variation was on average $10 \mathrm{~m}$, while the pitch was $3 \mathrm{deg}$, and both had long-range correlations. Non-repeatability of these parameters had a strong impact on the measured CSEM fields - an order of magnitude larger than the impact due to the 4-D effects in the reservoir (Fig. 5). Note also that in the previous section we considered non-repeatability of different acquisition parameters separately, while now CSEM data are generated assuming that all these parameters are changing "at once". The resulting combined effect of non-repeatability will thus be even stronger than for any individual plot in Fig. 5 .

We further assume that despite acquisition parameters have changed from survey to survey, they were measured accurately and the CSEM inversion was set up using their correct values. This situation describes very well the real acquisition scenario: while the water conductivity varies over time due to varying temperature and salinity, its depth profile can be measured accurately during the survey. The receiver landing position is difficult to predict due to uncertainties in the strength and direction of water currents, but it can be measured quite accurately afterwards using acoustic positioning systems. One can hope therefore that running inversions that "know" about the actual values of acquisition parameters can be a more promising method to detect 4-D effects than comparing raw CSEM data.

The top-left ATR map in Fig. 6 shows changes in transverse resistance between the base case and the $2^{\text {nd }}$ monitoring stage, for fully identical surveys. It is thus a copy of the ATR map in the $2^{\text {nd }}$ row and third column of Fig. 4, only using a denser color scale. We then regenerate CSEM data for the monitoring stage 2 assuming that acquisition parameters have changed as explained above. These data are inverted to produce a transverse resistivity map, which is again subtracted from the corresponding map obtained by inverting the base-case CSEM data with intact acquisition parameters. Their difference, that is the ATR map, is shown just below the "identical surveys" map in Fig. 6. Remarkably, these two top ATR maps look very similar to each other: one needs to work hard in order to find differences between them with a human eye. This is a direct visual illustration that survey non-repeatability should not be a problem in extracting 4-D CSEM response if the analysis is performed in model-domain, that is by inverting the data. At least, it is orders of magnitude smaller problem than for data-domain type of analysis where the 4-D response from the reservoir was totally masked by the non-repeatability effects (Fig. 5). In the top right image of Fig. 6 we present the difference between ATR maps for the "identical" and "non-repeatable" cases. The differences are so small that we had to compress the color scale by a factor of 10 to make them visible. Thus, the red color in a local region with the largest difference means that even there the difference is only $\sim 10$ per cent of the actual resistivity change. Both positive and negative differences represent an error in resistivity, therefore the analysis should consider the absolute value. By integrating this absolute difference over the map, one obtains 1.2 per cent of the integrated reservoir ATR. This number can be compared to 31 per cent reduction in the integrated reservoir ATR due to reservoir production at the monitoring phase 2. We can see that non-repeatability here has a 25 times smaller impact on inversion results than production-induced changes of resistivity in hydrocarbon-bearing layers. This is in a stark contrast to the analysis in data domain where acquisition non-repeatability affected EM data at least 10 times stronger than production-related changes in the reservoir. Note also that in real 4-D CSEM surveys the non-repeatability impact can be further reduced, for example by deploying receivers in a denser grid, by ensuring receiver position repeatability better than $50 \mathrm{~m}$ and by using detailed reservoir models for constraining EM inversions.

The two bottom rows of Fig. 6 help us clarify the reason for this dramatic progress in handling non-repeatability issues when going from the data-domain to the model-domain. Here we choose only one acquisition parameter to be allowed to vary between the surveys - the receiver position. Two cases are considered: (1) receivers are displaced by $50 \mathrm{~m}$ on average, but their positions are accurately measured and provided to inversion; (2) receivers are not displaced, but their positions in one of the two surveys are measured with an error of $3 \mathrm{~m}$ on average and these errors propagate to the inversion input. Here it is most instructive to focus on the right column of Fig. 6 and remember that all images there have the same color scale. As expected, displacing receivers by $50 \mathrm{~m}$ (middle image) has a smaller impact on inversion results than the same displacement accompanied by non-repeatability also in source parameters and water conductivity (top image). At the same time, a measurement error of only $3 \mathrm{~m}$ in receiver position (bottom image) distorts the inverted ATR maps to a much larger degree. We therefore conclude that a more serious problem in resolving subtle 4-D effects is the measurement errors during acquisition. Meanwhile, one can allow a significant variation of acquisition parameters from survey to survey without affecting the resolution as long as these variations are accurately measured.

\section{UNCERTAINTY IN BACKGROUND}

So far in all inversions the start model was built using the correct background resistivity from the "true" synthetic models. This is an overly optimistic approach since the background resistivity is never known precisely. At the same time, a 4-D study assumes a production setting, typically with numerous wells drilled in the area along with other geophysical studies. Hence, the knowledge of background resistivity should be fairly good, and one should not expect too large deviations between the inversion start model and reality. In this section we show how uncertainty in the background resistivity affects the results presented above.

The "true" background resistivities were smoothened to produce "imprecise" background resistivities for inversions. The smoothing length was $600 \mathrm{~m}$ in depth direction and $6000 \mathrm{~m}$ in both lateral directions. Fig. 7 shows slices of vertical background resistivity: the original one and after smoothing. The shown slices do not include the reservoir resistivities since the inversion start models are built assuming absence of hydrocarbons. The differences between the smoothened and original resistivity distributions look significant, especially, at the reservoir depth (below $2000 \mathrm{~m}$ ) where the geological layers are thinner. The bottom panel shows the impact of background smoothening on the CSEM data (again for the receiver marked by a circle in Fig. 2). The impact is considerable: it is well above the sensitivity threshold for a broad range of offsets and frequencies. Note also that our inversion setup implies that the background resistivity is not updated. It means that the errors in background resistivity of the start model will persist until the final model, the inversion will not be allowed to correct the background values when converging towards minimal data misfit. Meanwhile, the good news is that inversions of the base-case and the monitoring data will use the same incorrect background. Thus, one can hope for some error cancellations when analysing the resistivity differences between the two inversions. 


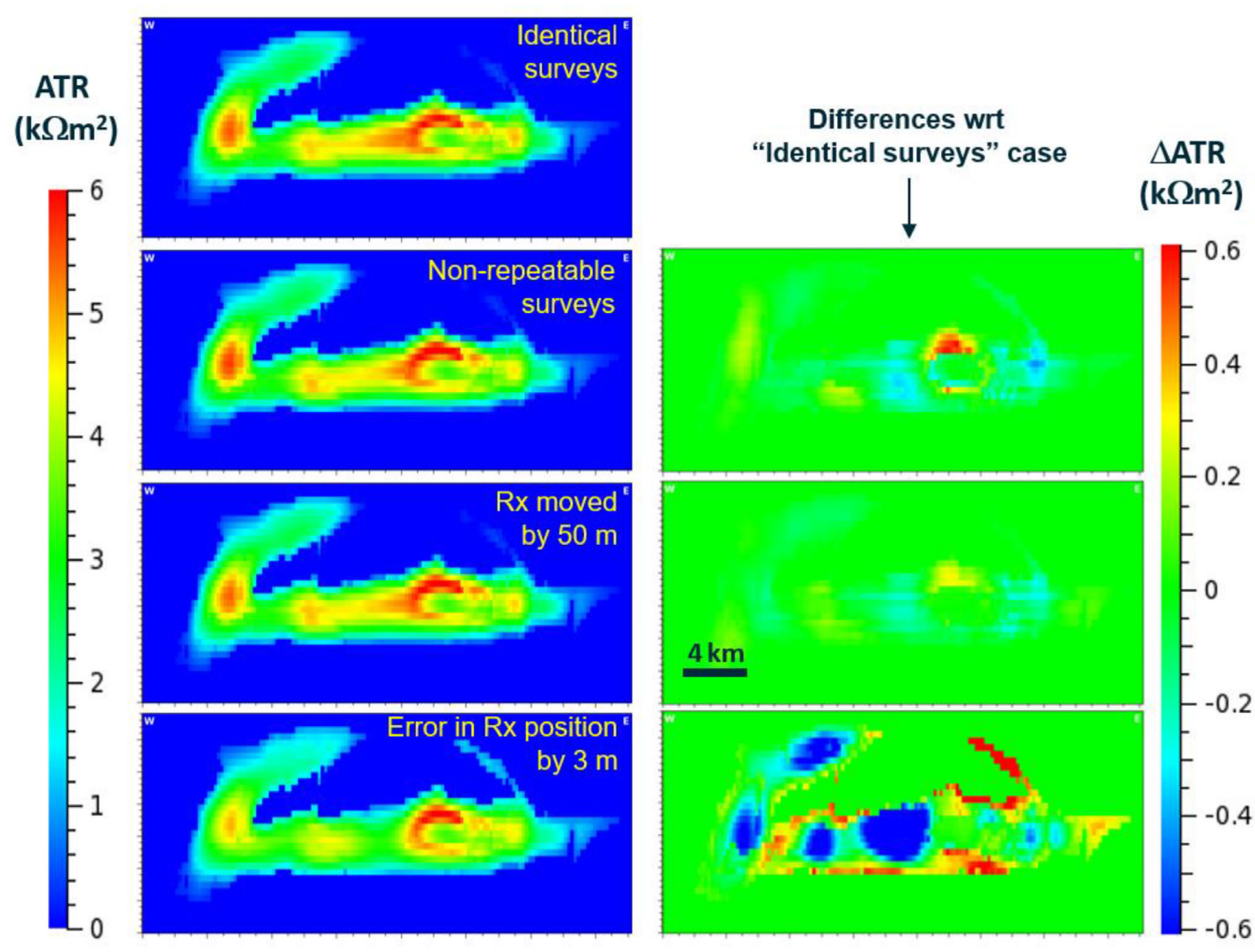

Figure 6. Negligible effect of non-repeatability in acquisition parameters on 4-D CSEM response in inversion results. Left: ATR maps - changes in transverse resistivity between the base and $2^{\text {nd }}$ monitoring stage. ATR map for non-repeatable surveys is as good as that for fully identical surveys (two top images). Small differences between them are highlighted on the right with an enhanced color-scale. Two bottom rows show that a small error $(3 \mathrm{~m})$ in receiver position damages inversion results much more than receiver displacement by $50 \mathrm{~m}$.

The inversion results assuming incorrect background resistivity are presented in Fig. 8. The left column shows ATR-maps of production-induced changes in resistivity, again for identical and for non-repeatable acquisition parameters. These two images are again very similar to each other implying that non-repeatability is not a serious problem for resolving 4-D effects. These two images are however clearly different from the corresponding two images for the correct background which are displayed as the top left panels of Fig. 6. To emphasize that, we present here, in the right column, the difference ATR-maps. The top-right map shows the impact of using imprecise background resistivity, and the bottom-right map shows the impact of having non-repeatable acquisition parameters. Obviously, inaccuracies in the background resistivity distribution lead to a much stronger distortion of the ATR-maps, that is reduce our ability to detect production-induced changes in resistivity. At the same time, variation of acquisition parameters between the base and monitoring surveys results in very minor distortions in ATRmaps. The magnitude of these distortions is of the same order as for the case of correct background (Fig. 6).

\section{DISCUSSION}

Though the presented resistivity maps give a very detailed information about the quality of the detected 4-D response, some useful quantitative insights can be obtained by integrating these maps over the reservoir area. Namely, we shall analyse the total productioninduced resistivity change $\Delta R$, defined as

$$
\Delta R=\int A T R d x d y=\int_{\substack{\text { voservoir } \\ \text { volume }}}\left(R_{\text {Base }}-R_{\text {Monit }}\right) d V
$$

Fig. 9 plots this total resistivity change $\Delta R$ extracted from inversions as a function of "true" $\Delta R$ found from synthetic models used to generate CSEM data fed into inversions. There were three such models for three monitoring stages, hence three different values of the "true" $\Delta R$ on the plot. For an ideal inversion, these two quantities should coincide, that is inversion should recover exactly 100 per cent of the reservoir resistivity, or in the 4-D case, exactly 100 per cent of resistivity reduction due to reservoir production. One can see from the plot that all inversions result in somewhat smaller $\Delta R$, that is the inversions here always underestimate the actual resistivity change. The underestimation factor is approximately 1.3 when the correct resistivity background is used. Similar underestimation factors have been observed earlier in 3-D CSEM studies, for example 1.28 for the Skrugard oil reservoir (Gabrielsen et al. 2013) and 1.1 for the Troll oil field (Morten et al. 2012). The good news is that this underestimation should not cause any problems when inverting field data. The underestimation factor can be estimated from synthetic inversions and then used to recalibrate inversions of field-data.

Unfortunately, it is not possible to compensate for imprecise knowledge of the background resistivity in a similar way (because 

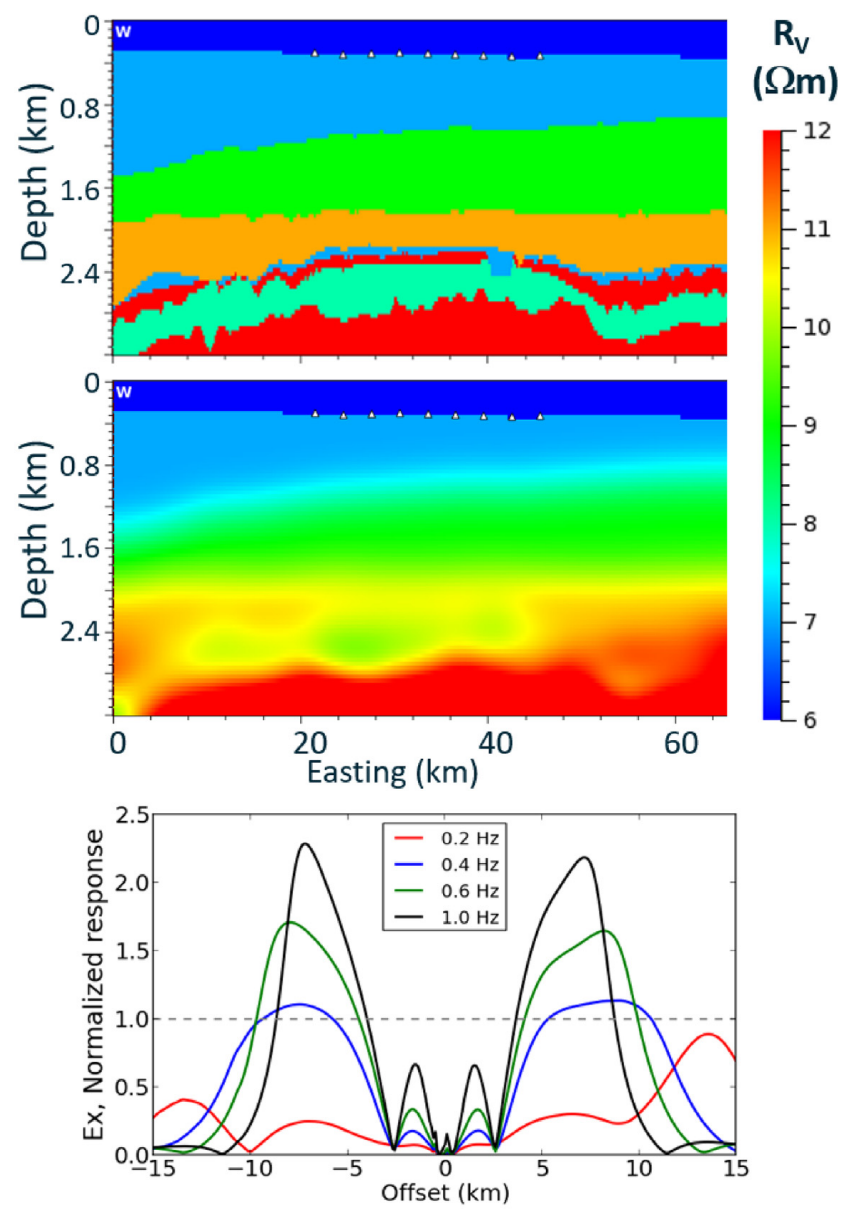

Figure 7. Top: True vertical background resistivity (without hydrocarbons). Middle: a smoothened background resistivity used in inversion. Bottom: impact of imprecise background knowledge on CSEM data, for a receiver marked in Figs 2 and 3c.

the precise resistivity is not known). In our example of extensively smoothened background, it results in approximately 25 per cent reduction of the total resistivity change, $\Delta R$. This is something to keep in mind for practical applications of 4-D CSEM. Besides, this is a serious motivation to develop a dedicated 4-D inversion tool that can take in both the base- and the monitoring CSEM data sets simultaneously and converge to a unique background resistivity and two different reservoir resistivity distributions. With such a tool the problem of constrained incorrect background will naturally disappear, while the resistivity variations will still be focused only to a small reservoir area, thus boosting inversion efficiency.

Fig. 9 also gives a visual answer to the central question of our study: the importance of acquisition non-repeatability for 4D CSEM. For the case of correct background, the effect of nonrepeatability is essentially invisible on the plot as the two lines corresponding to identical and non-repeatable cases almost coincide. The difference between them is only 0.44 per cent of $\Delta R$ of the full reservoir. For the wrong background, the difference is slightly larger, but still does not exceed 1.5 per cent. This is thus another indication that by performing 4-D analysis in the model domain one makes non-repeatability of acquisition parameters a rather minor issue, at least compared to the challenges of minimizing the measurement error and establishing the background resistivity.
Note also that the dependences of inverted $\Delta R$ vs true $\Delta R$ in Fig. 9 follow straight lines with a pretty good precision. This indicates absence of any visible non-linear effects in the inverted resistivities for early stages of production. This is good news for practical applications of 4-D CSEM as it may simplify analysis of inversion results and gives an additional confidence in the robustness of the inversion algorithm. Moreover, it probably means that our results obtained for relatively late production stages (17 per cent reduction in ATR for monitoring phase 1) may be transferrable to earlier production stages when potential benefits of time-lapse investigations can be very high. On the other hand, a significant reduction in ATR might occur very early in production due to strongly non-linear dependence of resistivity on hydrocarbon saturation.

In order to get more insights into 4-D CSEM feasibility, we ran an additional test for an early production stage where the oil-water contact has moved only by $5 \mathrm{~m}$ (for the monitoring stage 1 it has moved by $12.5 \mathrm{~m}$ ). In this case, 4-D changes in resistivity were very small, thus it was more challenging to extract quantitative and accurate information from inversion results. While the integral inverted resistivity stayed at 73.5-73.7 per cent of its true value for monitoring stages 1 and 2, here the ratio dropped to 67.2 per cent. The impact of non-repeatability in survey parameters has also become very strong: the absolute difference in the ATR integrated over reservoir's lateral extent amounted to almost 20 per cent of its production-induced changes.

Since we operate with very small differences in resistivity, of the order of 1 per cent, let us revisit the accuracy of the presented analysis. An advantage of a synthetic study is that we know how much noise is added to the data and can directly evaluate its impact on the inversion result. Namely, we can contaminate synthetic data with two different random realizations of the noise term, invert these two data sets and compare the resulting ATR maps. Taking the absolute value of their ATR differences and integrating over the reservoir area, gave us 2.1 per cent of the integrated reservoir ATR. This level of $\sim 2$ per cent sets the minimum limit on the ATR precision in the present study since all inverted synthetic data were contaminated with the same amount of noise. Therefore, we cannot really claim that the non-repeatability impact is 1.2 per cent, as computed above, because this value is below our ATR precision for the assumed noise level. We can only say that this impact cannot be much larger than 2 per cent of the integrated reservoir ATR. In fact, for the case of a smoothed background, the non-repeatability impact is slightly larger: 2.2 per cent of the integrated reservoir ATR instead of 1.2 per cent, but it is also mostly within the 2 per cent "precision limit".

The accuracy of the presented resistivity maps and $\Delta R$ computed from them looks better than one could have expected from Fig. 3 showing that the 4-D response for the 2nd monitoring stage is hardly above the data uncertainty. It usually means that the "imaging criterion" (Mittet \& Morten 2012) is not fulfilled, that is an inversion should not be able to image the corresponding changes in resistivity. Indeed, when we tried standard unconstrained inversions, it was essentially impossible to recover meaningful 4-D effects, because slight changes in resistivity were distributed throughout the computational domain. A dramatic improvement in the quality of inverted resistivity maps (and hence $\Delta R$ ) was achieved by running focused inversions that update resistivity only within the reservoir volume. This improvement is caused by effectively eliminating the nonuniqueness problem - a major inversions challenge. Similar findings have been reported by Zerilli et al. (2018) where "localized" inversions performed much better than "mesh-based" 

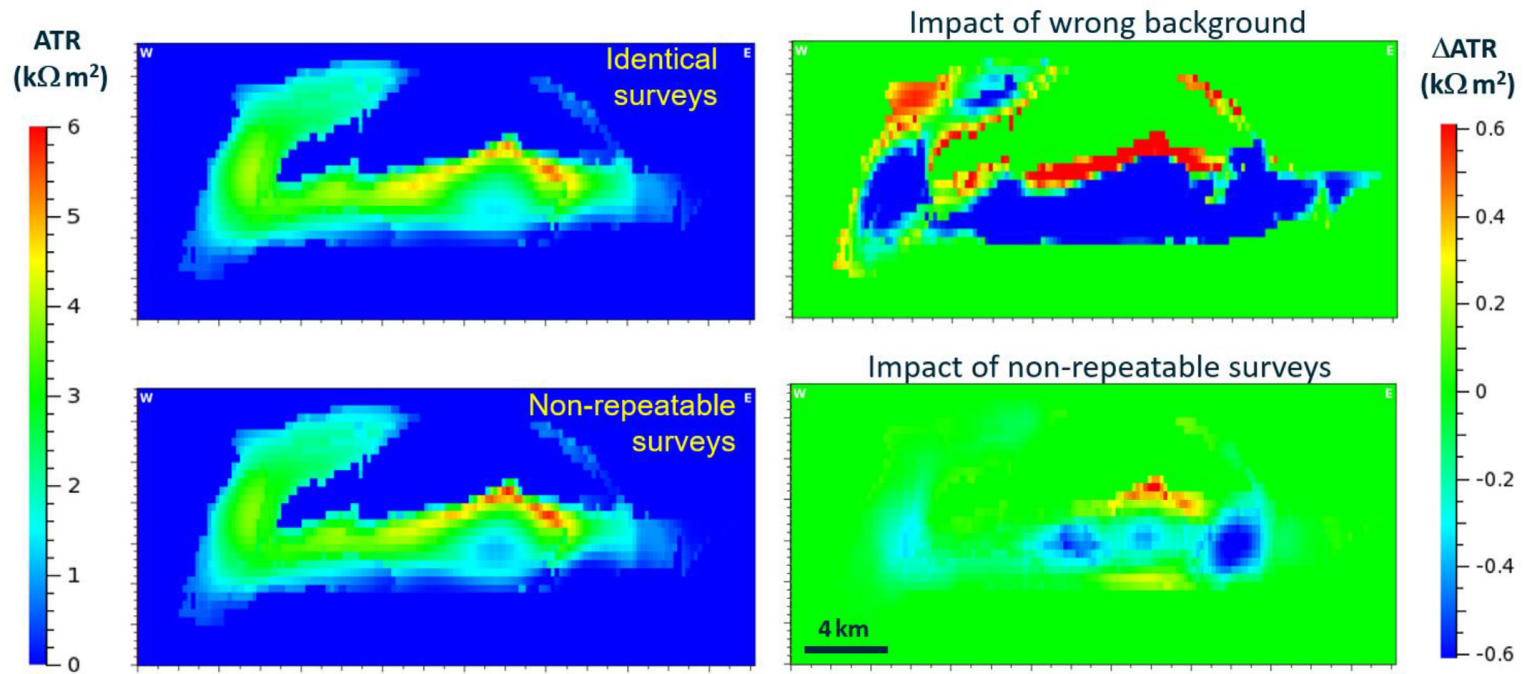

Figure 8. Inversion results based on imprecise background resistivity. Left: ATR-maps showing production-induced changes in inverted resistivity, for identical and non-repeatable surveys. Right: differences in ATR-maps caused by imprecise background (top) and by non-repeatability of acquisition parameters (bottom). Shown resistivity changes are between the base and the $2^{\text {nd }}$ monitoring stage.

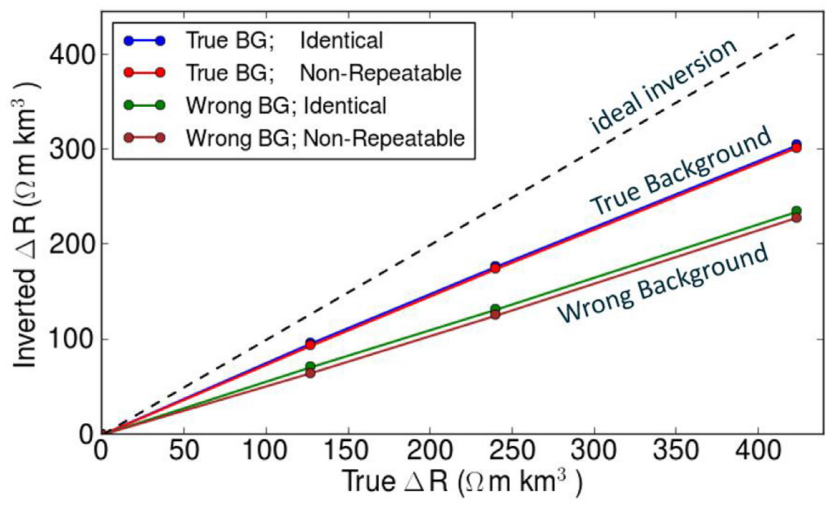

Figure 9. Production-induced change of resistivity integrated over reservoir: value obtained from CSEM inversions versus "true" value used to generate CSEM data. Three sets of points correspond to 3 monitoring stages. Dashed line corresponds to the ideal inversion that recovers 100 per cent of reservoir resistivity. Two upper and two lower lines correspond to inversions using correct and slightly incorrect background resistivity, respectively.

inversions. One can argue therefore that 4-D CSEM should in general have a better resolution than 3-D CSEM thanks to the fact that resistivity changes are localized within a small volume where production (or injections) takes place.

It has been assumed above that the mask defining the volume where inversion is allowed to update resistivity, coincides precisely with the reservoir volume. In a real setting, the reservoir outline is not known precisely, thus we ran an additional test using a slightly extended mask to account for uncertainty in the reservoir geometry. A few hundred meters were added in the lateral directions along the reservoir edges so that the new mask has become 10 per cent larger than the original one. It resulted in leakage of resistivity outside of the reservoir and consequently, less accurate inversion results. Namely it led to systematically lower average inverted resistivities. E,g., the total resistivity change $\Delta R$ for the monitoring phase 2, went down from 73.7 per cent of the true $\Delta R$ for the perfect mask to 68.3 per cent for the extended mask. Our main focus is however on non-repeatability and there were no big changes there: both with a perfect or with an extended mask, the non-repeatability impact was still on the level of ATR precision, 2 per cent.

We did not run additional tests for a mask that is extended in the vertical direction. Even though there always exists uncertainty in the depth placement of reservoir, it is less critical for CSEM studies because of the transverse-resistance equivalence principle. The principle says that it is mainly the thickness-resistivity product that determines the CSEM response of a resistive layer, and this is exactly the reason why our analysis is focused on ATR maps rather than 3-D distributions of resistivity. We have checked that the TR equivalence principle holds to an accuracy of better than 1 per cent if we increase the thickness and at the same time decrease anomalous resistivity of the current reservoir by 25 per cent each.

The assumed 1 per cent multiplicative noise and the noise floors of $10^{-16} \mathrm{~V} / \mathrm{Am}^{2}$ and $10^{-13} \mathrm{~m}^{-2}$ are quite small, and we decided to also run a few inversions on data contaminated with 3 per cent multiplicative noise and three times the additive noise. As a result, the data uncertainty increased by a factor of 3 throughout all offsets. For that case, according to Fig. 3, the 4-D effects should get below the resolution limit. Indeed, the quality of inversion results decreased, for example for the monitoring phase 2, inversions could recover only 56 per cent of the reservoir resistivity. However, it is not only the noise level that matters, but also how it relates to the target response. For example, if 4-D effects are expected to give 5-10 per cent change in CSEM fields, then a multiplicative noise of $\sim 3$ per cent should not be a serious problem.

An additional source of non-repeatability between the base and monitoring surveys is possible changes in the infrastructure, for example new pipelines and other seabed installations. They cannot be handled in the same simple way as variations, for example in water conductivity since information about resistive properties and geometry of such installations is often unavailable. Nevertheless, one should hopefully be able to discriminate changes in CSEM data due to production at the reservoir depth from changes caused by new seabed installations. In fact, a pipeline usually presents a 1-D conductor and can be effectively considered as a secondary EM source. At the same time, it has already been demonstrated by eigenparameter analysis as well as by inverting field CSEM data that at least in some cases it is possible to independently resolve the 

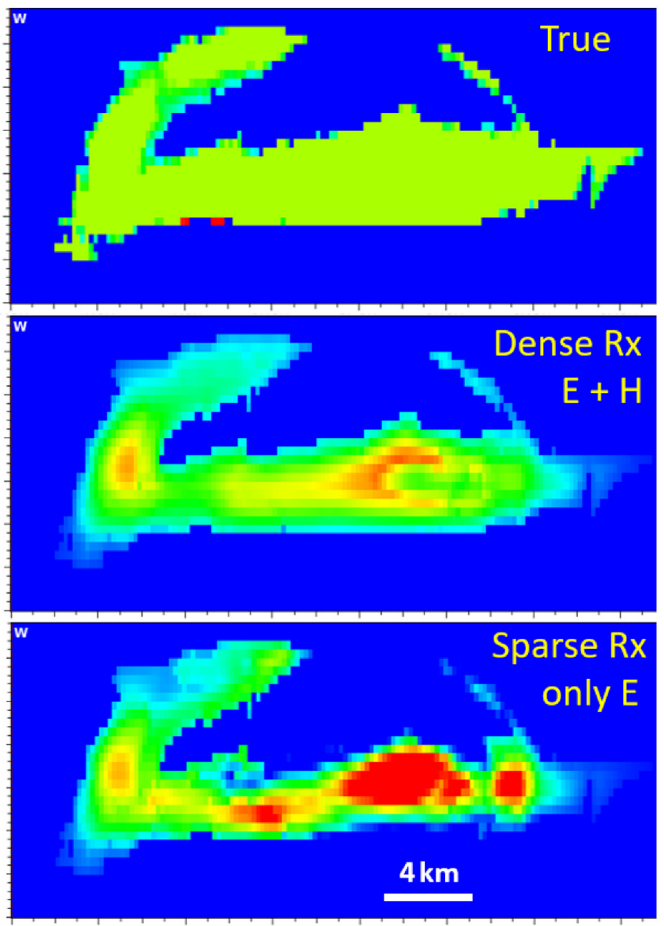

ATR $\left(\mathrm{k} \Omega \mathrm{m}^{2}\right)$

Figure 10. Impact of data density on quality of recovered 4-D response. Top: True ATR-map. Middle: Inverted ATR-map for double density of receivers, electric and magnetic field data. Bottom: Inverted ATR-map with sparser receivers and only electric field data.

subsurface resistivity and properties of EM source (Swidinsky \& Edwards 2011; Li \& Li 2017).

\section{IMPACT OF DATA DENSITY}

In this section we shall investigate how the quality of 4-D CSEM analysis is affected by data density, namely, (i) by including both electric and magnetic field data and (ii) by the density of the receiver grid at the seabed. So far we have used the receiver layout illustrated in Fig. 2 with $3 \mathrm{~km}$ spacing between receivers along the towing direction and $1 \mathrm{~km}$ spacing in the cross-direction. Besides, all our inversions so far have used both electric and magnetic fields. Let us now test two additional survey configurations. In the first one, the inline spacing between receivers is reduced from 3 to $1.5 \mathrm{~km}$, thus doubling the amount of data. In the second configuration, the receiver spacing remains $3 \mathrm{~km}$, but magnetic field data is excluded, that is inversions take as input only electric field data. Here, the amount of data is one half of the original setting, and the data type is modified. To summarize,

Configuration 1. More data: $1.5 \times 1 \mathrm{~km}$ receiver grid, $\boldsymbol{E}+\boldsymbol{H}$ fields Configuration 2. Less data: $3 \times 1 \mathrm{~km}$ receiver grid, only $\boldsymbol{E}$ field

ATR-maps showing the production-induced change in reservoir resistivity (for monitoring stage 2) are presented in Fig. 10 for both new configurations. They can be compared to the "true" ATR-map shown at the top of the Figure. It is clear that data density has a tremendous impact on the quality of ATR-map. Configuration 1 with denser receivers and both fields shows a very uniform distribution of resistivity in good agreement with the "true" ATR-map. Configuration 2 with sparser receivers and excluding magnetic data gives a less consistent map where ATR is strongly non-uniform and may locally deviate from its true values by more than a factor of two. For the original configuration with $3 \mathrm{~km}$ inline spacing and $\boldsymbol{E}+\boldsymbol{H}$ fields, the quality of the ATR-map (see Fig. 6, top image) lies between the new configurations 1 and 2. This implies that both factors —nser receivers, and inclusion of $\boldsymbol{H}$ data-contribute to improving the resolution of 4-D CSEM. Our results are thus consistent with findings of Granli et al. (2018) that a denser receiver and transmitter configuration can significantly improve the imaging resolution. In this section we have assumed identical survey parameters; investigations of the interplay between CSEM data density and non-repeatability effects are left for future work.

\section{FIELD DATA：WISTING}

Our conclusion about lower significance of non-repeatability effects for 4-D CSEM requires proper tests on field data. Such a test would imply acquisition of at least two CSEM surveys over the same area, preferably with the same survey layout. Since this would have been a rather expensive exercise, we instead present a preliminary field test based only on one CSEM survey. We split the acquired CSEM data into two non-overlapping subsets that can, to some degree, imitate two distinct acquisitions. Since both subsets were acquired at the same time, there will be no 4-D effects observed. Hence all differences in the two inverted resistivity distributions will stem only from different source (or receiver) positions in the two subsets. If the observed resistivity differences will be small, this may serve as another indication that a precise repeatability of the acquisition geometry is not that critical for 4-D CSEM applications.

The test CSEM study was carried out over the Wisting discovery made in the Norwegian Barents Sea in 2013 by operator OMV Norge, see Fig. 11(a). The reservoir is located in the Mid- Upper Jurassic, Realgrunnen Sub-group, at unusually shallow depth of merely $237 \mathrm{~m}$ below seabed (i.e. at $662 \mathrm{~m}$ TVD). The reservoir quality is excellent with high porosities and permeabilities. The Wisting reservoir was robustly identified even from the first CSEM survey over the area - a multiclient acquisition in 2008 - despite a rather sparse receiver grid. The excellent CSEM sensitivity here is partly due to extremely high resistivities in the oil-filled reservoir: in the well $\operatorname{logs}$ they reach values above $100 \mathrm{k} \Omega \mathrm{m}$, while the background resistivity is about $20 \Omega \mathrm{m}$. These high resistivities and low burial depth paved the way for a new 3-D CSEM survey over Wisting in 2014 - with a denser data coverage and much higher frequencies.

In the dense survey the receivers were deployed in a regular grid with spacing of $2 \mathrm{~km}$, while towlines were spaced only by $1 \mathrm{~km}$. More details about the 2014 CSEM survey and data interpretation can be found in Granli et al. (2017). In the present study we exclude a few receivers and towlines located farther from the Wisting reservoir, and use CSEM data for 59 receivers and 18 towlines. We further focus only on electric field data and choose frequencies of 1, 2, 4, 8, 10 and $16 \mathrm{~Hz}$ for inversion.

Our goal now is to split all the data from this survey into two nonoverlapping subsets that will be considered as two separate surveys with slightly different acquisition geometry. One simple approach is to divide all receivers into two groups by, e.g. taking every second receiver for each receiver line. The original survey with $2 \times 2 \mathrm{~km}$ receiver grid, will then convert into two repeated surveys with $2 \times$ $4 \mathrm{~km}$ receiver grid where each receiver was displaced by $2 \mathrm{~km}$ between the surveys. This approach is not very useful since receiver displacement by $2 \mathrm{~km}$ leads to very strong changes in the CSEM sensitivity and hence very different inversion results for such a shallow and compact target as Wisting. A more promising approach is to split CSEM data by source positions: here one can choose a 

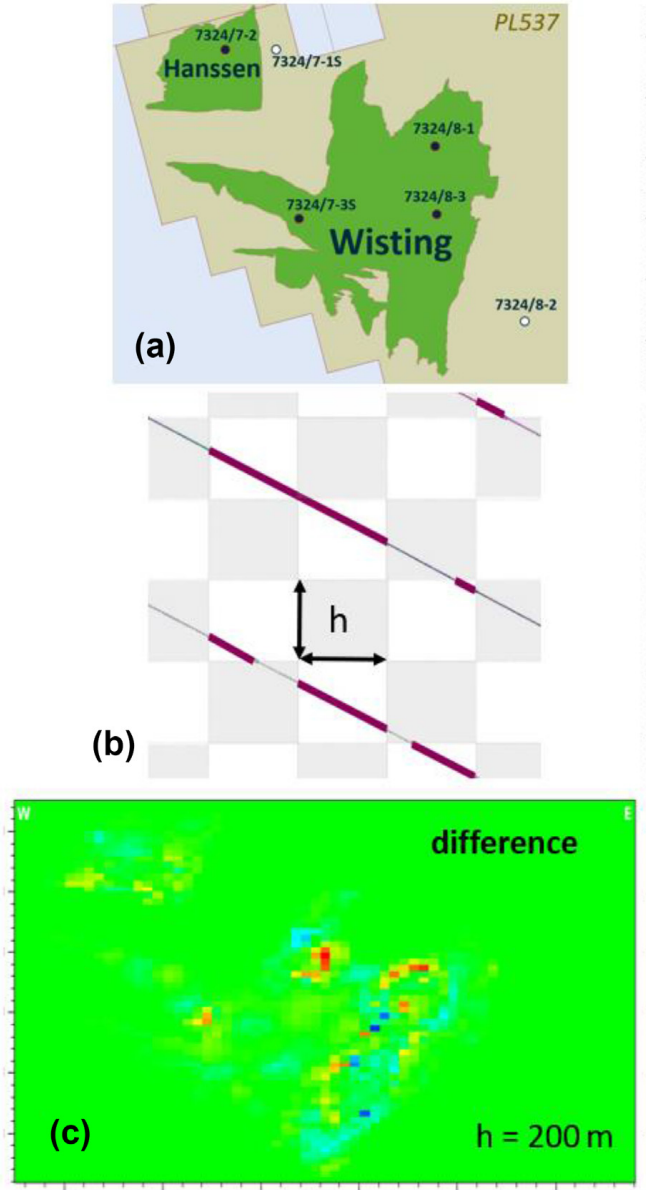

ATR $\left(k \Omega m^{2}\right)$
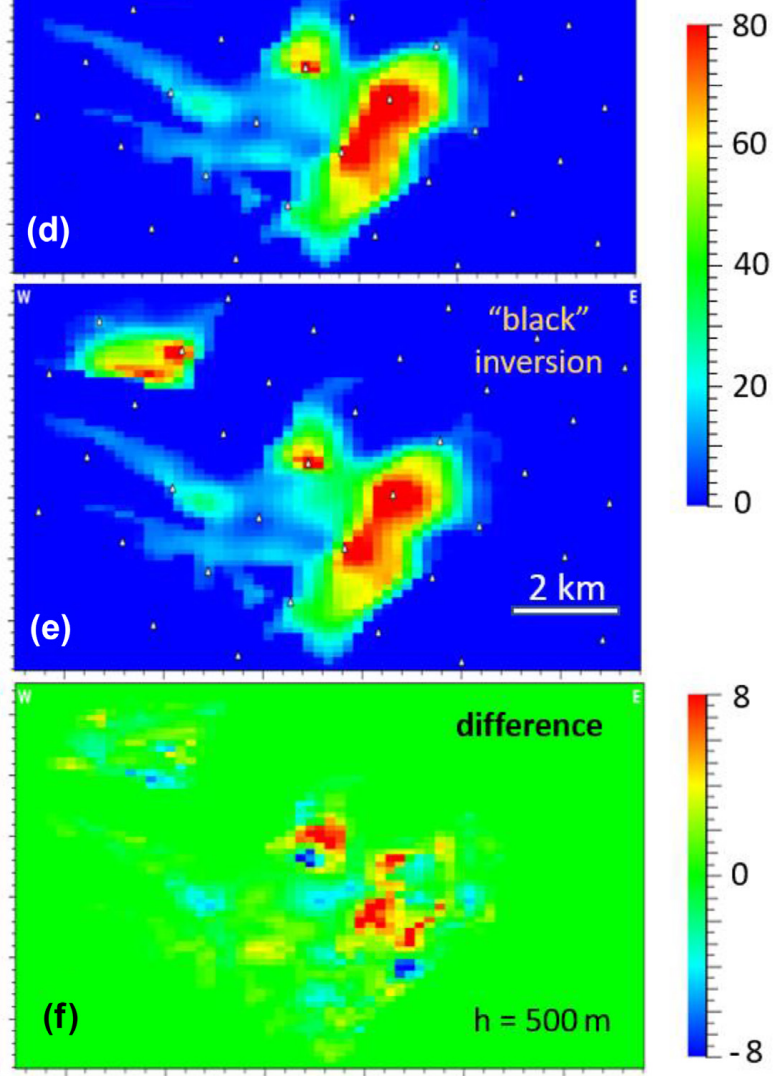

Figure 11. Impact of acquisition geometry on field data. (a) Wisting and Hanssen reservoirs in the Barents Sea. (b) Source positions along towlines are divided into "white" and "black" groups based on a chessboard pattern. (d,e) Resistivity maps obtained by inverting "white" and "black" CSEM data, respectively, for chessboard step $\mathrm{h}=500 \mathrm{~m}$. (c,f) Difference between resistivity maps for the "white" and "black" data on a compressed color scale, for h= $200 \mathrm{and} 500 \mathrm{~m}$.

smaller step since the source is moving continuously along the towlines. Moreover, to avoid aliasing in NS or EW directions, the split can be performed using a chessboard pattern, as illustrated in Fig. 11 (b). Source positions from "white" squares, indicated as thick segments of towlines, were grouped into a "white" survey, while positions from "black" squares (thin towline segments) formed a "black" survey. One can think of the "white" and "black" surveys as repeated acquisitions over the same area with a non-repeatability of the source positions given by the size of the chessboard squares. In principle, the size of black and white squares could have been chosen close to $10 \mathrm{~m}$ - to represent a realistic non-repeatability in source position for time-lapse studies. However, it cannot be smaller than the size of the FFT window used to transform the CSEM data into frequency domain, otherwise one may introduce correlations between data in the "white" and "black" subsets. This is a serious constraint since the typical FFT window is $\sim 100 \mathrm{~m}$. Therefore, we decided to conduct this study for two different chessboard sizes, 200 and $500 \mathrm{~m}$, to be able to see the trend of how this size impacts the obtained results.

3-D inversions were set up similarly to the procedure described above for the Snøhvit target, but with some modifications since we here deal with field data and do not know the "true" resistivity model. First the whole data set ("white" + "black") was inverted in order to determine the background resistivity model used for the following focused inversions. The start model had a linear resistivity gradient going from 11 to $20 \Omega \mathrm{m}$ for vertical resistivity $\left(R_{\mathrm{V}}\right)$ and from 4 to $8 \Omega \mathrm{m}$ for horizontal $\left(R_{\mathrm{H}}\right)$, starting from the seafloor and down to $4 \mathrm{~km}$ TVD. Slightly extended NPD outlines for the Wisting and Hanssen reservoirs were used to define the "reservoir region", while the rest was considered as background. As expected, anomalously high resistivities produced by the inversion were confined within the outlined reservoirs. The depth interval of the "reservoir region" was 480-720 m. In contrast to Granli et al. (2017), we did not use seismic interpreted surfaces or appraisal wells to set inversion constraints. Thus, our background resistivity is much less accurate than one would normally expect in a real 4-D project, which presents an additional challenge for the focused inversions described below.

At the next step we ran two focused inversions on the "white" and the "black" subsets of data. These focused inversions were allowed to update resistivity only within the "reservoir region", while the background resistivity was fixed. The start models for the focused inversions were obtained from the final model of the unconstrained inversion after overwriting the "reservoir region" with resistivities $R_{\mathrm{V}}=10 \Omega \mathrm{m}$ and $R_{\mathrm{H}}=2 \Omega \mathrm{m}$ that are slightly lower than typical background values. Focused inversions were building up resistivity from those small values towards $\mathrm{k} \Omega \mathrm{m}$ range. The ATR maps computed over the reservoir depth interval for the two inversions are 
presented in Figs 11(d,e). Here we show results for the $500 \mathrm{~m}$ size of chessboard squares, that is for stronger "non-repeatability". The anomalous resistivity is defined with respect to the start model and integrated over the depth interval of 480-720 m. Note that these ATR maps are not meant for reservoir interpretation as they were obtained without any geological inputs and used down-sampled CSEM data. For high-resolution resistivity maps over Wisting and nearby area see analysis in Granli et al. (2017) and Granli et al. (2018) that combines several geophysical methods.

We are interested in analysing differences between ATR maps (d) and (e) produced by the "white" and "black" inversions. The maps look very similar to each other: the shapes and strength of both Wisting and Hanssen anomalies are almost identical. Panel (f) shows the difference between them on a 10 times denser color scale. The ATR difference strongly varies from location to location, and one can see clusters of opposite polarities (red and blue), with cluster size being comparable to $500 \mathrm{~m}$. One may argue therefore that the differences in ATR stem from different patterns of CSEM sensitivity for the white and black survey layouts. Higher CSEM sensitivity in a particular part of the reservoir usually means that the inversion will produce higher resistivity in that part. Let us now quantify the ATR differences by following the same procedure as for the synthetic case. Integrating the absolute value of the ATR difference over the whole area gives us an average difference of 5.8 per cent of the total integrated ATR. It is larger than the corresponding number, 1.2 per cent, for the synthetic Snøhvit case, probably because the source position variation increased from $\sim 10 \mathrm{~m}$ to $\sim 500 \mathrm{~m}$. Indeed, rerunning the Wisting inversions with a chessboard size of 200 instead of $500 \mathrm{~m}$ reduces the corresponding difference from 5.8 to 3.5 per cent. It also reduces the size of the characteristic red/blue clusters, as can be seen by comparing panels (f) and (c). The reduction in size down to $\sim 200 \mathrm{~m}$ is consistent with the above interpretation based on CSEM sensitivity that should also vary on a $\sim 200 \mathrm{~m}$ scale. These red and blue clusters, corresponding to enhanced and reduced ATR, effectively cancel each other when integrating over the reservoir area. As a result, the integrated anomalous resistivity, $\Delta R$, defined by eq. (2), differs by less 0.2 per cent between the "white" and "black" surveys. These two numbers, 3.5 per cent and 0.2 per cent characterize the impact of non-repeatability in our pseudo-4-D field study.

Several factors make our pseudo-4-D study different from a realistic 4-D scenario. First, the receiver positions/orientations and water conductivity were identical for our "white" and "black" surveys, though in a real 4-D study these will be different, thus adding to nonrepeatability effects. Second, source positions for the "white" and "black" surveys were different by 200 or $500 \mathrm{~m}$, while in a realistic 4-D setup one will aim at repeating exactly the same source trajectory, and the corresponding differences will be orders of magnitude smaller, probably $\sim 10 \mathrm{~m}$. The corresponding non-repeatability effects will then also be much smaller, as evident from the trend observed by comparing $200 \mathrm{~m}$ and $500 \mathrm{~m}$ results. The impact of non-repeatability should be further reduced by applying geological constraints on CSEM inversions (e.g. from seismic data) similar to the approach of Granli et al. (2017).

\section{CONCLUSION}

Repeatability of survey parameters in time-lapse CSEM studies is not a critical issue if 4-D effects are analysed in the model domain, that is by employing the inverted resistivity models. A synthetic study on a Snøhvit gas field demonstrates that even significant variations of source position and orientation, receiver position and seawater conductivity between the base and monitoring surveys have a marginal impact on the resolution of 4-D CSEM. Namely, random errors in inverted resistivity caused by non-repeatability effects are comparable to resistivity changes in the reservoir upon producing 1.2 per cent of its hydrocarbon volume.

Our conclusion holds even for the challenging case when differences in the recorded EM fields induced by non-repeatability of acquisition parameters are at least 10 times stronger than the 4-D response-differences in EM fields due to changes in the reservoir resistivity. In that case 4-D effects can still be detected by inverting of CSEM data, and the impact of non-repeatability on the inverted resistivity maps will be at least 10 times smaller than the resolved time-lapse changes in the reservoir resistivity.

A more important factor that may affect the 4-D CSEM sensitivity is accurate measurements of acquisition parameters. It is shown that a $3 \mathrm{~m}$ error in receiver position leads to a much larger error in the extracted 4-D response than a $50 \mathrm{~m}$ displacement of receivers between the base and monitoring surveys. Another important factor for the quality of 4-D CSEM analysis turns out to be a good knowledge of the background resistivity distribution. Denser receiver grids and use of both electric and magnetic field data also significantly improve the spatial resolution of CSEM to 4-D responses.

Our conclusions are further supported by inverting field data acquired over the Wisting oil discovery. Here the impact of nonrepeatability was estimated by comparing differences in resistivity maps obtained by inversion of two non-overlapping subsets of the same CSEM survey. Non-repeatability only in the source position, but at an exaggerated scale of $200 \mathrm{~m}$, leads to fine-scale variations in inverted resistivity with 3.5 per cent amplitude and only 0.2 per cent variations in the total resistivity.

\section{ACKNOWLEDGEMENTS}

The authors wish to thank EMGS for permission to publish the results, as well as Amir Babakhani, Ole Martin Pedersen, Jan Petter Morten, Martin Hansen, John Reidar Granli and Max Meju for comments and discussions.

\section{REFERENCES}

Alcocer, J.A.E., García, M.V., Soto, H.S., Baltar, D., Paramo, V.R., Gabrielsen, P. \& Roth, F., 2013. Reducing uncertainty by integrating 3D CSEM in the Mexican deep-water exploration workflow, First Break, 31, 75-70.

Andreis, D. \& MacGregor, L., 2011. Using CSEM to monitor production from a complex 3D gas reservoir - a synthetic case study. Leading Edge, 30(9), 1070-1079.

Babakhani, A., 2015, Repeatability and detectability requirements for 4D CSEM, Master Thesis. University of Oslo, Norway.

Baltar, D. \& Roth, F., 2013. Reserves estimation methods for prospect evaluation with 3D CSEM data. First Break, 31, 103-110.

Bhuyian, A.H., Landrø, M. \& Johansen, S.E., 2012.3D CSEM modeling and time-lapse sensitivity analysis for subsurface $\mathrm{CO}_{2}$ storage, Geophysics, 77(5), E343-E355.

Black, N., Wilson, G.A., Gribenko, A.V., Zhdanov, M.S. \& Morris, E., 2011. 3D inversion of time-lapse CSEM data based on dynamic reservoir simulations of the Harding field, North Sea, in Proceedings of the 81th Annual International Meeting, SEG, Expanded Abstracts, pp. 666-670, SEG.

Colombo, D. \& McNeice, G.W., 2013. Quantifying surface-to-reservoir electromagnetics for waterflood monitoring in a Saudi Arabian carbonate reservoir, Geophysics, 78(6), E281-E297. 
Constable, S., 2010. Ten years of marine CSEM for hydrocarbon exploration, Geophysics, 75(5), 75A67-75A81.

Gabrielsen, P.T., Shantsev, D.V. \& Fanavoll, S., 2012. 3D CSEM for hydrocarbon exploration in the Barents Sea, in Proceedings of the 5th EAGE Saint Petersburg International Conference \& Exhibition on GeosciencesMaking the Most of the Earth's Resources, Expanded Abstracts, C002, EAGE.

Gabrielsen, P.T., Abrahamson, P., Panzner, M., Fanavoll, S. \& Ellingsrud, S., 2013. Exploring frontier areas using 2D seismic and 3D CSEM data, as exemplified by multi-client data over the Skrugard and Havis discoveries in the Barents Sea, First Break, 31, 63-71.

Girard, J.-F., Coppo, N., Rohmer, J., Bourgeois, B., Naudet, V. \& SchmidtHattenberger, C., 2011. Time-lapse CSEM monitoring of the Ketzin (Germany) $\mathrm{CO}_{2}$ injection using 2xMAM configuration, Energy Proc., 4, 3322-3329.

Granli, J.R., Hafslund Veire, H., Gabrielsen, P.T. \& Morten, J.P., 2017. Maturing broadband 3D CSEM for improved reservoir property prediction in the Realgrunnen Group at Wisting, Barents Sea, in Proceedings of the 87th Annual International Meeting, SEG, Expanded Abstracts, pp. 2205-2209, SEG.

Granli, J.R., Daudina, D., Robertson, S.C., Morten, J.P., Gabrielsen, P.T. \& Sigvathsen, B., 2018. Applying high-resolution 3D CSEM and seismic for integrated reservoir characterization, in Proceedings of the 88th Annual International Meeting, SEG, Expanded Abstracts, pp. 949-953, SEG.

Hanssen, P. et al., 2017. The next generation offshore CSEM acquisition system, in Proceedings of the 87th Annual International Meeting, SEG, Expanded Abstracts, pp. 1194-1198, SEG

Kang, S., Kyubo, N., Jee, S.S. \& Joongmoo, B., 2015. mCSEM inversion for $\mathrm{CO} 2$ sequestration monitoring at a deep brine aquifer in a shallow sea, Expl. Geophys., 46(3), 236-252.

Landrø, M., 2001. Discrimination between pressure and fluid saturation changes from time-lapse seismic data, Geophysics, 66(3), 836-844.

Li, G. \& Li, Y., 2017, Joint inversion for transmitter navigation and seafloor resistivity for frequency-domain marine CSEM data, J. appl. Geophys., 136, 178-189.

Liang, L., Abubakar, A. \& Habashy, T.M. 2012, Joint inversion of controlledsource electromagnetic and production data for reservoir monitoring, Geophysics, 77, ID9-ID22.

Lien, M. \& Mannseth, T., 2008. Sensitivity study of marine CSEM data for reservoir production monitoring, Geophysics, 73(4), F151-F163.

Løseth, L.O., Wiik, T., Olsen, P.A. \& Hansen, J.O., 2014. Detecting Skrugard by CSEM - Prewell prediction and postwell evaluation, Interpretation, 2(3), SH67-SH78.

Mittet, R. \& Morten, J.P., 2012. Detection and imaging sensitivity of the marine CSEM method, Geophysics, 77, E411-E425.

Morten, J.P., Roth, F., Karlsen, S.A., Timko, D., Pacurar, C., Olsen, P.A., Nguyen, A.K. \& Gjengedal, J., 2012. Field appraisal and accurate resource estimation from 3D quantitative interpretation of seismic and CSEM data, Leading Edge, 31, 447-456.

Nguyen, A.K., Nordskag, J.I., Wiik, T., Bjørke, A.K., Boman, L., Pedersen, O.M., Ribaudo, J. \& Mittet, R., 2016. Comparing large-scale 3D GaussNewton and BFGS CSEM inversions, in Proceedings of the 86th Annual International Meeting, SEG, Expanded Abstracts, pp. 872-877, SEG.

Orange, A., Key, K. \& Constable, S., 2009. The feasibility of reservoir monitoring using time-lapse marine CSEM, Geophysics, 74, F21-F29.

Park, J., Sauvina, G. \& Vöge, M., 2017. 2.5D inversion and joint interpretation of CSEM data at Sleipner $\mathrm{CO}_{2}$ storage, Energy Proc., 114, 3989-3996.
Patzer, C., Ritter, O. \& Tietze, K., 2017. Time-lapse CSEM inversion using focusing regularization techniques for reservoir monitoring, in $S E G$ Technical Program Expanded Abstracts, pp. 5870-5874, SEG

Perez, L.A.S., Panzner, M. \& Soto, H.S., 2020. Reconstructing salt geometry using 3D CSEM data, First Break, 38(3), 43-47.

Qu, S. \& Verschuur, D.J., 2017. Simultaneous joint migration inversion for accurate time-lapse analysis of sparse monitor surveys, in Proceedings of the First EAGE Workshop on Practical Reservoir Monitoring, 6-9 March 2017, Amsterdam, The Netherlands.

Salako, O., MacBeth, C. \& MacGregor, L., 2015. Potential applications of time-lapse CSEM to reservoir monitoring, First Break, 33(4), 35-46.

Shahin, A., Key, K., Stoffa, P. \& Tatham, R., 2012. Petro-electric modeling for CSEM reservoir characterization and monitoring, Geophysics, 77, E9-E20.

Shantsev, D.V., Roth, F. \& Ramsfjell, H., 2012. Surface towing versus deep towing in marine CSEM, in Proceedings of the 82th Annual International Meeting, SEG, Expanded Abstracts, 0317, SEG.

Shantsev, D.V., Nerland, E.A., Babakhani, A. \& Gelius, L.J., 2018. Relaxed repeatability requirements for 4D marine CSEM: inversion study, in Proceedings of the 88th Annual International Meeting, SEG, Expanded Abstracts, 934-938, SEG.

Streich, R., 2016. Controlled-source electromagnetic approaches for hydrocarbon exploration and monitoring on land, Surv. Geophys., 37, 47-80.

Swidinsky, A. \& Edwards, R.N., 2011. Joint inversion of navigation and resistivity using a fixed transmitter and a towed receiver array: a transient marine CSEM model study, Geophys. J. Intl., 186, 987-996.

Tietze, K., Ritter, O. \& Veeken, P., 2015. Controlled-source electromagnetic monitoring of reservoir oil saturation using a novel borehole-to-surface configuration, Geophys. Prospect., 63, 1468-1490.

Tietze, K., Ritter, O., Patzer, C., Veeken, P. \& Dillenand, M., 2019. Repeatability of land-based controlled-source electromagnetic measurements in industrialized areas and including vertical electric fields, Geophys. J. Int., 218, 1552-1571.

Wang, Z. \& Gelius, L.-J., 2010. Electric and elastic properties of rock samples: a unified measurement approach, Petrol. Geosci., 16, 171-183.

Wirianto, M., Mulder, W.A. \& Slob, E.C., 2010. A feasibility study of land CSEM reservoir monitoring in a complex 3-D model, Geophys. J. Int., 181, 741-755.

Zach, J.J., Bjørke, A.K., Støren, T. \& Maaø, F., 2008. 3D inversion of marine CSEM data using a fast finite-difference time-domain forward code and approximate Hessian-based optimization, in Proceedings of the 78th Annual International Meeting, SEG, Expanded Abstracts, pp. 614618, SEG.

Zach, J., Frenkel, M.A., Ridyard, D., Hincapie, J., Dubois, B. \& Morten, J.P., 2009. Marine CSEM time-lapse repeatability for hydrocarbon field monitoring, in Proceedings of the 79th Annual International Meeting, SEG, Expanded Abstracts, pp. 820-824, SEG.

Zerilli, A., Labruzzo, T., Golfre' Andreasi, F., Menezes, P.D.T.L., Crepaldi, J.L.S. \& Alvim, L.D.M., 2018. Realizing 4D CSEM value on deep water reservoirs - the Jubarte case study, in Proceedings of the 80th EAGE Conference and Exhibition, Expanded Abstracts, Tu E 11, SEG.

Zhdanov, M.S., Endo, M., Black, N., Spangler, L., Fairweather, S., Hibbs, A., Eiskamp, G.A. \& Will, R., 2013. Electromagnetic monitoring of CO2 sequestration in deep reservoirs, First Break, 31(2), 71-78.

Ziolkowski, A., Parr, R., Wright, D., Nockles, V., Limond, C., Morris, E. \& Linfoot, J., 2010. Multi-transient electromagnetic repeatability experiment over the North Sea Harding field, Geophys. Prospect., 58, 1159 1176. 